\title{
Radiative cooling of size-selected gas phase clusters
}

\author{
Piero Ferrari ${ }^{1}$, Ewald Janssens ${ }^{1}$, Peter Lievens ${ }^{1, *}$, Klavs Hansen ${ }^{2,3, *}$ \\ ${ }^{1}$ Quantum Solid State Physics, Department of Physics and Astronomy, Faculty of \\ Science, KU Leuven, 3001 Leuven, Belgium \\ ${ }^{2}$ Center for Joint Quantum Studies and Department of Physics, School of Science, \\ Tianjin University, 92 Weijin Road, Tianjin 300072, China \\ ${ }^{3}$ Department of Physics, University of Gothenburg, 41296 Gothenburg, Sweden
}

$$
07 / 10 / 2019,15: 31
$$

Keywords: Clusters, Thermal radiation, Recurrent fluorescence

*peter.lievens@kuleuven.be

*klavshansen@tju.edu.cn

Predicted almost forty years ago, the radiation from thermally populated excited electronic states has recently been recognized as an important cooling mechanism in free molecules and clusters. It has presently been observed from both inorganic clusters and carbon-based molecules in molecular beams and ion storage devices. Experiments have demonstrated that many of these systems radiate at rates approaching microsecond time scales, and often with a distinct dependence on the precise number of atoms in the system. The radiation acts as a strongly stabilizing factor against both unimolecular decay and thermal electron emission. In astrophysical context, radiative cooling provides a mechanism to dissipate internal energy in star-forming processes, and stabilizes molecules selectively in the circumstellar medium. The consequences of an active radiative cooling channel for nanoparticle production will likewise favor special sizes in nonequilibrium formation processes. In this review, the radiative cooling of clusters is presented and illustrated with examples of experiments performed on small carbon, metal, and semiconductor clusters, and on PAH molecules. The experimental and theoretical techniques used are discussed, together with the consequences of radiative cooling on size-to-size stability patterns of clusters. 


\section{Contents}

1 Introduction $\quad 3$

2 Thermal decay channels of clusters $\quad 5$

3 Detection of thermal radiation $\quad 10$

3.1 Direct and indirect detection . . . . . . . . . . . . . . . . . 10

3.2 Non-radiative $1 /$ t decay . . . . . . . . . . . . . . . . . . 12

3.3 Radiative quenching of unimolecular decay . . . . . . . . . . . . . . . 19

4 The boundary between large and small photon energies 24

5 The effect of radiation on abundances $\quad 28$

5.1 Non-radiative abundances . . . . . . . . . . . . . . . . . . 28

5.2 Radiative cooling and abundances . . . . . . . . . . . . . . . 30

$6 \quad$ Vibrational cooling versus recurrent fluorescence 32

6.1 Decays of carbon-based clusters . . . . . . . . . . . . . . . . . 32

6.2 Radiative cooling rates of metal and semi-metal clusters . . . . . . . . . 35

$\begin{array}{lll}7 & \text { Comments on the theoretical status } & 37\end{array}$

8 Conclusions and outlook $\quad 38$

9 Acknowledgements $\quad 39$ 


\section{Introduction}

The properties of clusters, i.e. particles composed of a countable number of atoms, can differ significantly from those of the corresponding bulk material and will often show a strong size dependence for a number of properties [1-3]. This is particularly clear for optical properties, which critically depend on the specific electronic structure, whether described in terms of single, molecular-like transitions or collective excitations in the form of surface plasmon excitations [4-8]. Also thermal properties, most importantly the heat capacity, depend on size due to the limited number of degrees of freedom. This size effect is particularly important in experiments on isolated clusters where equilibration with an external heat bath is absent.

Thermal radiation from clusters must therefore be expected to be heavily influenced by finite size effects. The subject was first investigated during the eighties and nineties, on clusters composed of carbon and of niobium, produced with both broad size and excitation energy distributions [9-16]. In these experiments, where emitted photons were detected directly, spectra resembling black-body radiation within the measured visible spectral range were observed. Also experiments on size-selected species were performed [17, 18], although often the radiative cooling was investigated indirectly, without detecting any photons [19-22]. Direct detection of photons emitted from small, size-selected clusters

was achieved only recently, and so far only for two clusters, $\mathrm{C}_{4}^{-}$[23] and $\mathrm{C}_{6}^{-}$[24], both in experiments performed with an ion storage ring.

Both experiments with and without mass selection suggest that the emission of radiation from clusters can proceed from thermally populated electronically excited states. The indications are either the energy of observed photons or the high values of the indirectly measured radiative cooling rates. Fig. 1 illustrates the idea by which this emission happens in the scenario of the original prediction of the effect [25]. After absorption of a photon, the energy can either be re-emitted as a fluorescent photon or, in a competing process, dissipated in a radiationless process [26-29], by coupling to the vast number of vibrational states that exist at even moderate excitation energies. The motion from the initial excitation into the entropic vibrational manifold, known as internal conversion (IC), proceeds via some system-specific combination of Born-Oppenheimer surface dynamics and possibly intersystem crossings. At the end of this sequence, the system returns to the electronic ground state from which it may radiate vibrationally but obviously not electronically. Such vibrational radiative transitions in the electronic ground state have been observed in trap experiments, see for example [30,31], and is well established.

The return of the system to the initial excited state, or to any optically active excited 


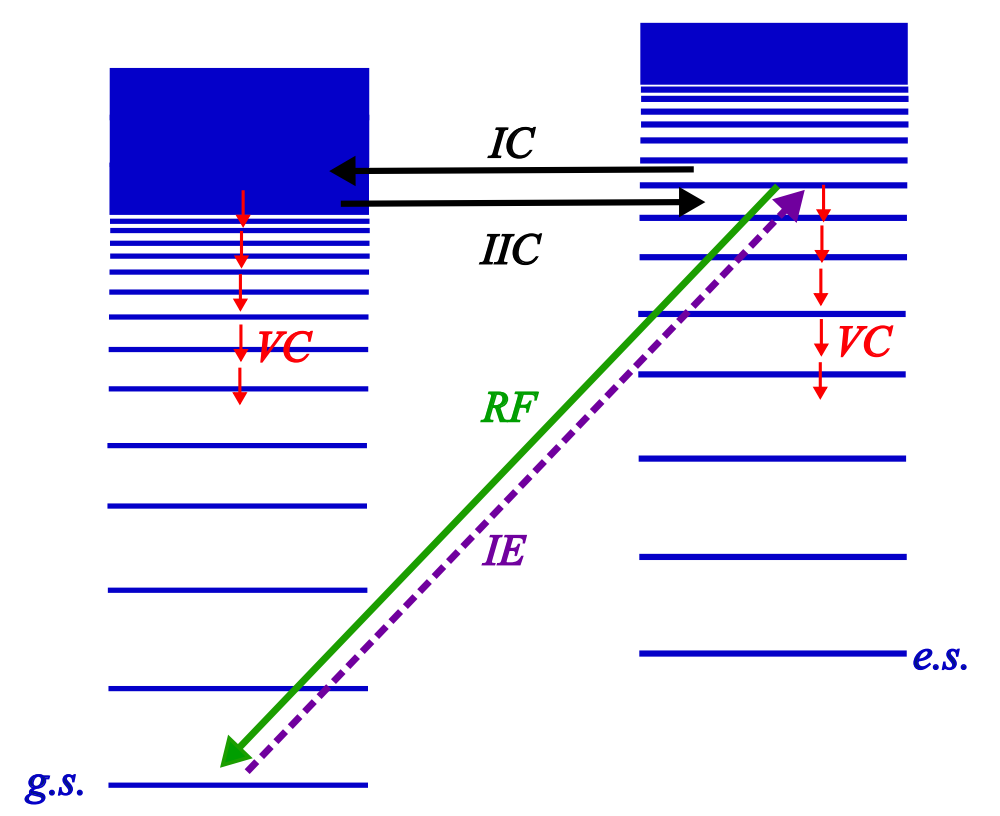

Figure 1: Schematic diagram of the main transitions in a molecular systems with a ground state (g.s.) and low-lying excited electronic state (e.s.). The arrows represent the processes of initial excitation (IE), internal conversion (IC), vibrational radiative cooling (VC), inverse internal conversion (IIC) and recurrent fluorescence (RF). The absorption of the photon from the ground state is shown as a dashed line as a reminder that the mechanism is not restricted to the specific excitation of photon absorption.

electronic state is, however, also possible by detailed balance. This process is denoted Inverse Internal Conversion (IIC), and for the same reasons that a photon can be absorbed into this excited state, a photon can be re-emitted from it.

The process, responsible for the thermal emission of visible and near-infrared photons, was initially considered in $[25,32,33]$ as arising after absorption of a photon. Because the theoretical predictions were associated with photon emission after energy dissipation, which was until then considered the ultimate equilibrated state of the system, the process was named recurrent fluorescence. However, by its nature of a statistical process, the presence and characteristics of IIC are independent of the precise mode of excitation of the molecule or cluster. In particular, it does not require that the initial excitation is optical. An example of infrared excitation converted to an electronic excitation is presented in [34].

The processes where the absorption of a photon is the method of initial excitation have been considered quantum versions of a Poincaré process where a system returns to its initial state [33]. Poincaré's calculation showed that even though an isolated (classical) 
system will return arbitrarily close to an initial state, this recurrence time is extremely long for a macroscopic system. So long, in fact, that the system in practice will never return to it and that the monotonous growth of entropy predicted by Boltzmann will therefore hold for all practical purposes. For smaller systems, however, the recurrence time is much reduced and can become so short that it reaches experimentally observable values. The analogy between IIC and a Poincaré recurrence should not be taken too far, though. In particular, the populations of large systems will not be suppressed with the size of the phase space, but rather be determined by the average excitation energy per degree of freedom. For this reason, recurrent processes must be expected to be much more common than the long period between prediction and discovery suggests.

The phenomenon is likely to be most relevant for the relatively strongly bound atomic clusters, because these may be able to sustain the elevated internal energies needed for the processes, and less relevant for molecular clusters, with their weak van der Waals or hydrogen bonds. No molecular clusters have yet been found to radiate by the RF mechanism. RF is inherently a high excitation energy phenomenon, and it appears most clearly when the molecules and clusters are as highly excited as the experimental time scales allow. Typical sizes investigated at the moment cover the range from very small clusters, such a tetramers, up to systems composed of some tens of atoms, while cooling times range from just a few microseconds (for RF) up to milliseconds (for VC). For $\mathrm{Au}_{9}^{+}$, for example, a radiative time constant of only $1.3 \mu \mathrm{s}$ has been observed. The principles discussed here, however, are broader valid and values outside those ranges may occur in specific situations.

\section{Thermal decay channels of clusters}

For gas phase clusters studied under high vacuum conditions, whether in molecular beams, ion traps or in ion storage rings, reactions are restricted to decays that cause irreversible energy loss. Different types of decays very often occur in parallel, shaping the observable dynamics of thermal photon emission in an essential way, and the basic features of these decays will therefore be outlined here.

Energy in excess of the lowest activation energy of any decay channel will ultimately be dissipated. The possible processes are emission of atoms, small molecules or larger moieties in a usual fragmentation process [35], electron emission [36-39], and photon emission, as illustrated in Fig. 2. For multiply charged clusters also a fission process, analogous to nuclear fission, can occur [40, 41], whereby charged fragments are emitted, resulting in a charge separation. Although such processes can also occur thermally activated, they 
have not been studied with respect to photon emission and will therefore not be discussed further here.

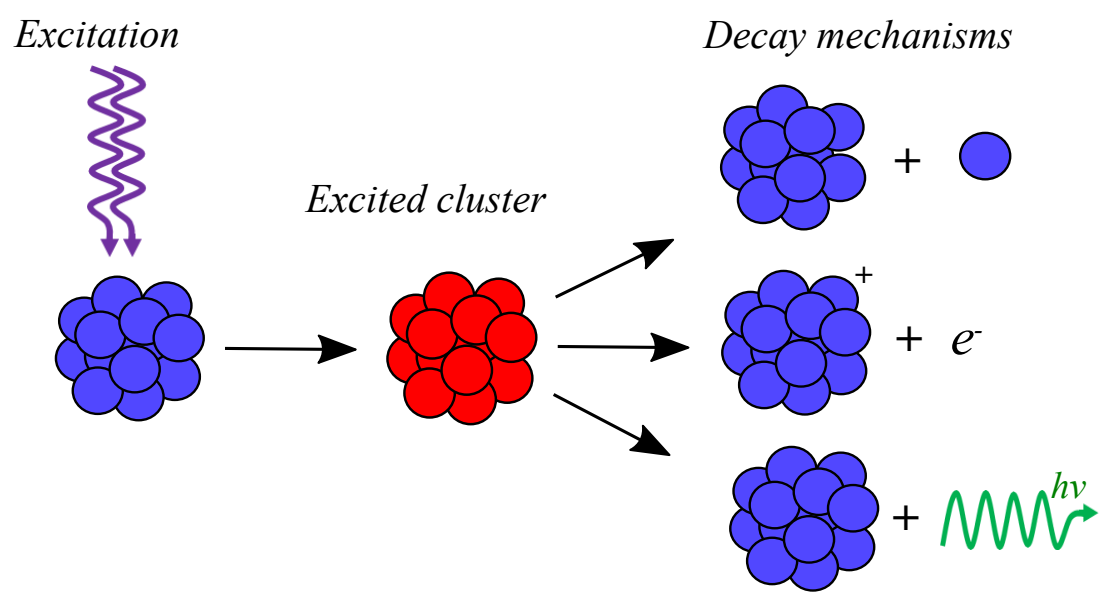

Figure 2: Schematic representation of the three mechanisms by which excited clusters decay. From top to bottom: fragmentation, electron emission and radiative cooling.

All of the three decay channels can in the simplest approximation be characterized by a frequency factor and an activation energy. The branching ratios of the channels depend critically on the numerical values of the frequency factors and on the activation energies associated with each channel. The competition between fragmentation, electron emission and radiation is exemplified in Fig. 3, showing a typical dependence of the decay rate constants on the internal energy $E$, of the cluster.

Expressions for these rate constants can be derived by detailed balance considerations [46]. For photon emission, the relative population of the emitting electronic state is given by its statistical weight, i.e. the number of vibrational and other states that can be characterized as belonging to the photon emitting configuration, relative to the total number of states at the given energy [47]. This ratio of level densities is modified by the stimulated emission. An analysis shows that the photon emission rate constant, $k_{N, \mathrm{rad}}$, from such an excited state is, expressed in terms of the Einstein $A$-coefficient, equal to [39]

$$
k_{N, \mathrm{rad}}(E)=A \frac{\frac{\rho_{N}(E-h \nu)}{\rho_{N}(E)}}{1-\frac{\rho_{N}(E-2 h \nu)}{\rho_{N}(E-h \nu)}},
$$

where $\rho_{N}$ is the density of states (DOS), for the cluster size $N$, and $h \nu$ the photon energy.

The only assumption made in the derivation of Eq. (1), apart from the statistical Ansatz, is that the photo-absorption cross section does not change after the emission of a single photon [46]. For not too small systems this will be a reasonable assumption 


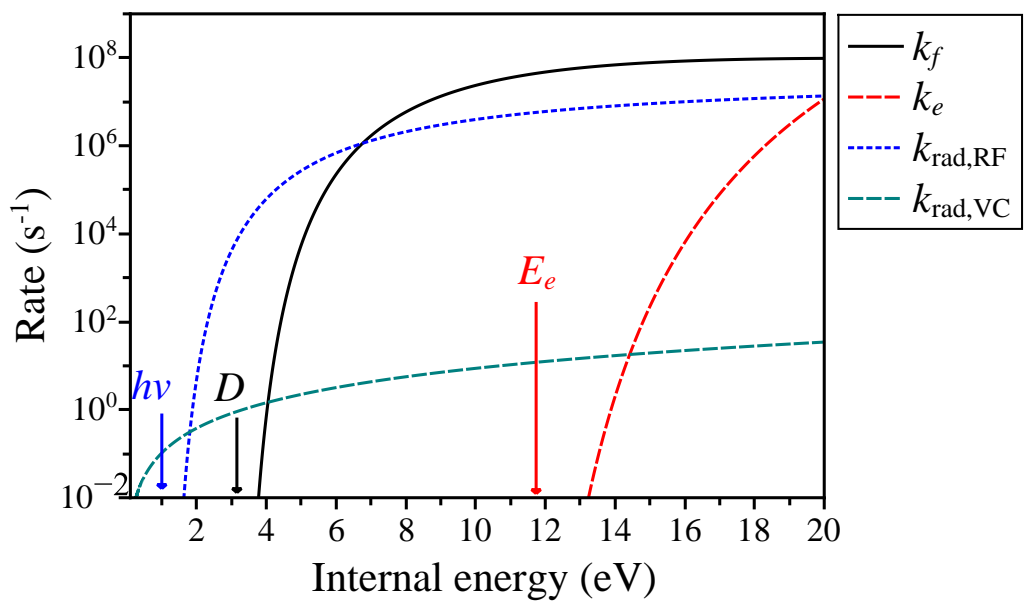

Figure 3: Rates of fragmentation, electron emission and radiation as a function of internal energy, exemplified by the nine-atom cationic gold cluster, $\mathrm{Au}_{9}^{+}$. The rates of fragmentation and radiation are calculated using input parameters from Refs. [42, 43]. The crossing point of the photon emission curve $\left(k_{\mathrm{rad}, \mathrm{RF}}\right)$ with the dissociation rate constant for dimer emission $\left(k_{f}\right)$ has been determined experimentally. To calculate the rest of the curve for $k_{\text {rad,RF }}$, the arbitrary value $h \nu=1.2 \mathrm{eV}$ was used. A smaller photon energy would make the curve flatter and vice versa. The rate of ionization is calculated with the ionization energy, $E_{e}$, from Ref. [44], corrected with a charging energy to give the relevant second ionization energy. Level densities are calculated with the BeyerSwinehart algorithm [45], assuming harmonic oscillators and frequencies calculated with DFT.

when the photon emission competes with a unimolecular decay, because total excitation energies will usually be much higher than a photon energy, and more so the larger the system is. For emission below the unimolecular threshold, however, this is not necessarily a good approximation and one may need to apply a system-specific analysis.

Some aspects of Eq. (1) are worth noting. First, that the activated nature of the process is indicated by the presence of ratios of level densities of the same cluster size and charge state, but evaluated at different energies. Secondly, we note that the term involving the ratio of level densities in the denominator of the equation, $\rho_{N}(E-2 h \nu) / \rho_{N}(E-h \nu)$, which appears as the consequence of stimulated emission, is evaluated at a different energy than the ratio in the numerator. This is a direct consequence of detailed balance $[39,46]$. Any negative energies that may appear in the level densities, there or in the numerator, are easily taken into account by setting the values of $\rho$ to zero for these energies. 
The photon emission rate constant due to vibrational transitions comes with the special feature that emission can occur from levels at different degree of excitation of the same, optically active mode, as long as the effective temperature is high enough to populate more levels than the lowest vibrationally excited state. The oscillator strengths associated with the levels of a single vibrational mode are proportional to the energy of the emitting level in the harmonic approximation. This makes the cross section energy-dependent. Introduction of this systematics into the general photon rate equation gives the vibrational photon emission rate constant for the vibrational mode $m$ [48]

$$
k_{N, m}(E)=A_{m} \sum_{n=1}^{\left[E / h \nu_{m}\right]} n \frac{\rho_{N}^{\prime}\left(E-n h \nu_{m}\right)}{\rho_{N}(E)}
$$

where $\rho$ is the total density of states and $\rho^{\prime}$ the density of states with mode $m$ excluded, and the square brackets in the upper limit of the summation indicate the integer part of the number. $A_{m}$ and $h \nu_{m}$ are the emission coefficient and vibrational quantum energy of mode $m$, respectively. Usually the summand has effectively vanished at values of $n$ far below the upper limit of the sum, and this can then be set to infinity without loss of precision. This facilitates analytical and numerical manipulations of the result, but is not a necessary step.

As photon emission is frequently measured by its effect on statistical, unimolecular dissociation by loss of atoms or small molecules, such rate constants must therefore also be considered. They are generally given approximately as (see e.g. chapter 5 of Ref. [46])

$$
k_{N, \Delta N, f}(E)=\omega_{N, \Delta N} \frac{\rho_{N-\Delta N}\left(E-D_{N, \Delta N}\right)}{\rho_{N}(E)},
$$

in which $\omega_{N, \Delta N}$ is the frequency factor of the channel, $\rho_{N-\Delta N}$ and $\rho_{N}$ are the level densities of the clusters indicated by the subscripts, and $D_{N, \Delta N}$ is the dissociation energy of the fragmentation channel $M_{N} \rightarrow M_{N-\Delta N}+M_{\Delta N}$. The detailed balance rate constant are particularly useful for clusters. Other expressions are in use, such as the RRKM theory and phase space theories. Most of the differences between these expressions are manifested in the precise value of the frequency factors in Eq. (3). In Eq. (3), and the one for electron emission given below, the factor multiplying the ratio of level densities is set to a size-dependent but energy-independent constant. This does not hold rigorously and much effort has gone into the description of these energy dependencies in the past [35]. However, for the application of the equations over the limited energy intervals relevant here it is a good approximation to set this factor constant. To avoid obscuring the resulting equations with unnecessary mathematical clutter, this will be done here. 
An expression similar to Eq. (3) can be written for the process of thermal electron emission. This is known as thermionic emission when it occurs from bulk surfaces, and this term has also been adopted for the process in clusters. The frequency factor is different from that of a unimolecular decay, mainly because of the different translational kinetic energy phase space factors of an electron compared with an atom or a small molecule. A second difference is the activation energy for electron emission which is the electron binding energy $\left(E_{e, N}\right)$, i.e. the ionization energy or the electron affinity, depending on the initial charge state of the particle. A third difference is the level densities involved, which for electron emission correspond to clusters of the same size, but different charge states [39]. The expression for the rate constant is

$$
k_{N,(q), e}(E)=\omega_{N,(q)} \frac{\rho_{N,(q+1)}\left(E-E_{e, N}\right)}{\rho_{N,(q)}(E)},
$$

where the level densities refer to the charge states $q, q+1$ of the parent and the product cluster, respectively.

An important consequence of the different energy dependence and frequency factors of the different types of emission rate constants is that usually a single type of decay will dominate at a given time. Over the limited time ranges where two or, very rarely, three curves cross, decays will be observed to occur in parallel.

The pattern seen in Fig. 3 with emission of massive particles (dimers in this case) at high excitation energies and of photons at low energies is a general feature for all materials and molecules. The reason is that the frequency factors for atomic/electron emission are much larger than the $A$-coefficients for photon emission. The former have values of $10^{15}$ $\mathrm{s}^{-1}$ or larger [46]. In contrast, the $A$-coefficients are typically on the order of $10^{9} \mathrm{~s}^{-1}$ or lower, and they will in any case be limited by the Thomas-Reiche-Kuhn sum rule on the dipole oscillator strength [49]. Photon emission will therefore not be a competitive channel at high excitation energy. At lower energy, a reduced activation energy for the process, viz. the photon energy, will compensate for this large difference in frequency factors and make photon emission competitive. Another consequence of the smaller activation energy for photon emission is that the variation of the photon emission rate constant is weak compared with the rate constant for emission of atoms and electrons. 


\section{Detection of thermal radiation}

\subsection{Direct and indirect detection}

The direct measurement of photons emitted from mass selected clusters has been accomplished in the two cases mentioned: $\mathrm{C}_{6}^{-}[24]$ and $\mathrm{C}_{4}^{-}$[23]. In these experiments, beams were produced in a hot source and injected into an electrostatic storage ring [50-52], and a photomultiplier tube detected photons emitted by the clusters. In a separate experiment, the clusters were laser excited geometrically separated and hence also time-wise separated from the photon detector, and the emitted photons detected analogously to those produced from source-heated clusters. The main result from the work on $\mathrm{C}_{6}^{-}$is shown in Fig. 4. In panel (a), the measured photon yields for $\mathrm{C}_{6}^{-}$are shown, with three

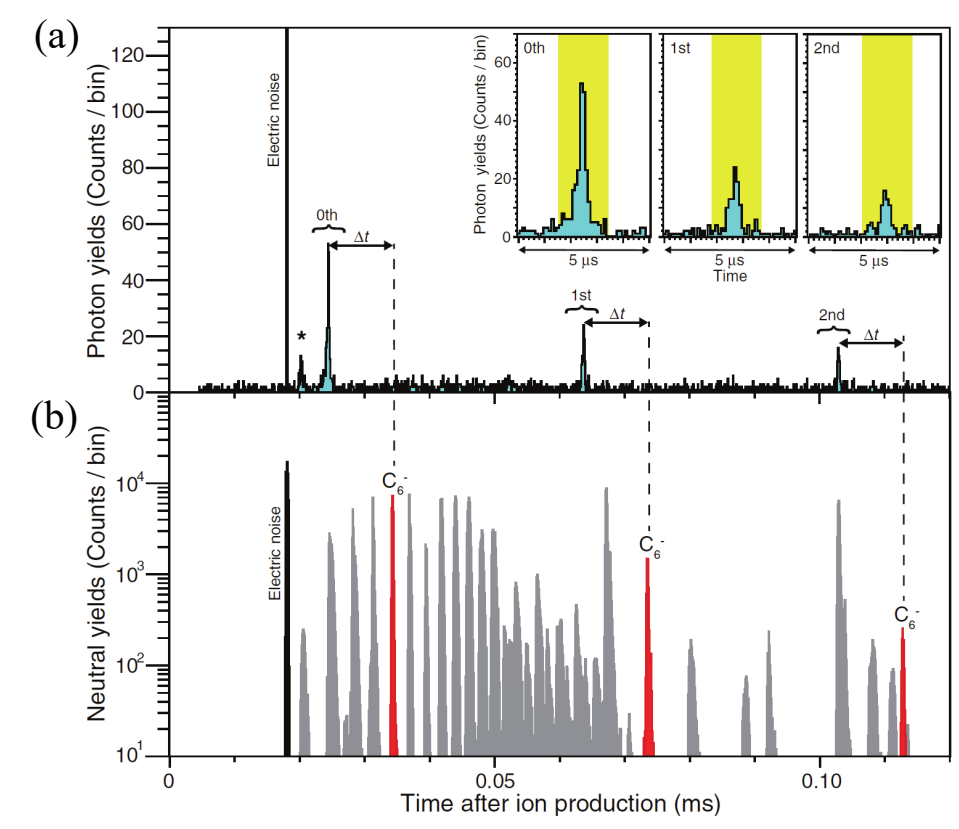

Figure 4: (a) Temporal profile of the photon yield after the $\mathrm{C}_{6}^{-}$ion production in the source. (b) Temporal profile of the neutral products. The time delay $(\Delta t)$ between the photon signals and the corresponding neutral product represents the flight time of a neutral fragment between the photon detector and the neutral particle detector. Reprinted with permission from [24]. Copyright (2016) by the American Physical Society.

distinct peaks corresponding to the times where the clusters were passing in front of the photodetector. Panel (b) confirms the identification of $\mathrm{C}_{6}^{-}$as the source of the detected photons, showing the coincidence between ions passage and photon detection. 
The effort required for such measurements is substantial, and alternatives techniques are highly desirable. Methods to detect radiation without observing the emitted photons have therefore been developed, historically before the direct detection. The general idea behind these experiments is to measure the influence of photon emission on another channel that is easier to monitor, i.e. the unimolecular loss of atoms, of small fragments or thermally emitted electrons. The idea was used in [19], where the influence of radiative cooling was used to explain the time dependence of decay of cationic fullerenes by $\mathrm{C}_{2}$ emission. It was applied soon after on thermionic emission data of fullerenes [20] and smaller carbon clusters [53], and again on cationic fullerenes [54].

Pump-probe experiments of the influence of radiation on competing unimolecular channels is the most direct method to measure radiative cooling with this strategy. In such pump-probe experiments, clusters or molecules are one-photon excited to some energy at or below the energy that will give an appreciable amount of unimolecular decay on the experimental time scale. A probe laser pulse, from which also only a single photon is absorbed, is then fired with a variable delay. In the absence of radiation, yields and time constants measured after the second photon will be independent of the pump-probe delay. Any change in e.g. measured unimolecular rate constants indicates the presence of radiation.

The first such experimental determination of an energy dependent radiation rate was reported in [55], where the pump-probe scheme was used to study the radiative cooling of $\mathrm{V}_{13}^{+}$clusters stored in a Penning trap. The stored ions were equilibrated to room temperature with a buffer gas to an excitation energy of $E_{i}=0.53 \mathrm{eV}$, after which two laser pulses of tunable wavelengths were used for single photon excitation in each laser pulse. The sum of the two photon energies was kept constant at $7.71 \mathrm{eV}$. Absorption of a photon from the pump pulse excited the clusters to an energy of $E_{0}=E_{i}+h \nu_{\text {pump }}$ which did not produce any observable dissociation on the measured time scale. If a probe photon of energy $h \nu_{\text {probe }}$ is absorbed with no or little pump-probe delay, clusters undergo fragmentation with some observable rate constant, $k_{f}$, in the process $\mathrm{V}_{13}^{+} \rightarrow \mathrm{V}_{12}^{+}+\mathrm{V}$. This situation is illustrated in the top frame of Fig. 5a, with generic rates of fragmentation (full blue line) and radiation (dashed red line) shown vs. internal energy. With a pumpprobe delay of $\Delta t$, say, clusters have time to cool radiatively, resulting in a lower internal energy, $E_{0}^{*}$, at the time the probe laser is fired. This situation is represented at the bottom panel of Fig. 5a. As a consequence of the cooling, the fragmentation induced by the absorption of the second photon proceed at the lower rate constant $k_{f}^{*}$. This rate constant is determined mass spectrometrically, as a function of time delay.

The data showed a clear decrease in the measured rate constant with increasing $\Delta t$. 
Fitting an exponential function gave the rate of radiative cooling for this photon energy. Repeating the procedure with different photon energies then allowed a quantification of the cooling rate as a function of internal energy, as shown in Fig. 5b (reproduced from $[55])$.
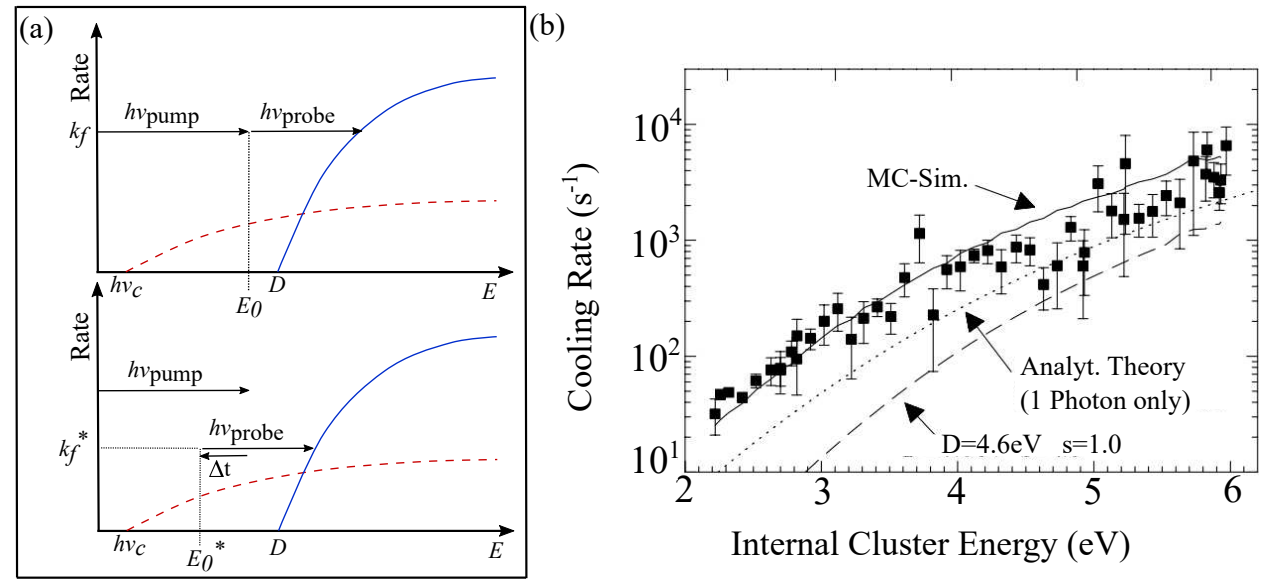

Figure 5: (a) Diagrams illustrating the experimental determination of the energy dependent photon emission rate of the $\mathrm{V}_{13}^{+}$cluster. See main text for details. (b) Measured photon emission cooling rates of $\mathrm{V}_{13}^{+}$, as a function of internal energy. Panel (b) reprinted with permission from [55]. Copyright (1999) by the American Physical Society.

\subsection{Non-radiative $1 / \mathrm{t}$ decay}

The simplest method to quantify radiative cooling is to measure the influence of radiation on unimolecular decay rates (the term 'decay rate' refers to the number of decays per unit time, as opposed to the rate constant, which is the quantity that appears in the exponential decay). The initial excitation may be provided by the production process in the source or by post-production, non-resonant laser excitation. As mentioned these excitation methods are equivalent in statistical processes.

The detection of radiation by its influence on a competing process of unimolecular nature, without direct detection of the photons emitted or application of a pump-probe scheme as above, would at first glance appear to run into a fundamental problem. Both competing processes, photon emission and unimolecular decay, are assumed thermal. This means that they both have an exponential time profile when decaying out of a state with some specific energy. Appearing in parallel, the combined effect of the two channels is to 
decrease the population of the excited state as

$$
P=P_{0} \mathrm{e}^{-t\left(k_{a}+k_{p}\right)}
$$

where $k_{a}$ is the unimolecular rate constant and $k_{p}$ the photon emission rate constant, assuming for the sake of argument that the emission of a single photon quenches any further unimolecular decay. The corresponding observable unimolecular decay rate is

$$
R=P_{0} k_{a} \mathrm{e}^{-t\left(k_{a}+k_{p}\right)}
$$

Although it is relatively easy to measure unimolecular decays and to determine the sum $k_{a}+k_{p}$ from such data, it is usually not experimentally feasible to assign any value specifically to $k_{p}$.

The fact that the competing channel method nevertheless works is due to the huge difference between the parameters that enter the rate constants for unimolecular decays vs. those of photon emission. This makes it possible to circumvent the problem by modifying one of the two exponential decays. This can be done within a statistical description of both decays. An added advantage is that the protocol, described in detail below, is also experimentally easy to implement. Under specific, but still fairly easily realisable conditions, the resulting unimolecular decay rate will have a $1 / t$ dependence in the absence of radiation [56].

To understand the mechanism behind this conversion, consider first the situation where only a single unimolecular decay, e.g. fragmentation, occurs with any significant amplitude in an ensemble of clusters, and where the internal energy distribution of this ensemble is broad. A schematic representation of this situation is given in Fig. 6, in which the vertical axis represents internal energy, and $E_{0}$ is the ground state.

Irrespective of the width of the energy distribution, the ensemble will decay with a rate, $R(t)$, given by an integration over the range of internal energies:

$$
R(t)=\int_{0}^{\infty} g(E, t) k(E) \mathrm{d} E
$$

where $g(E, t)$ is the population of the excited clusters at time $t$ and energy $E$. We have used the common symbol $k$ for the non-radiative rate constant in this equation to emphasize that Eq. (7) is valid for fragmentation $\left(k_{f}\right)$ as well as for electron emission $\left(k_{e}\right)$. In statistical decays, populations decrease exponentially due to the destructive decay by fragmentation or electron emission, i.e.

$$
\frac{\mathrm{d} g(E, t)}{\mathrm{d} t}=-k(E) g(E, t)
$$




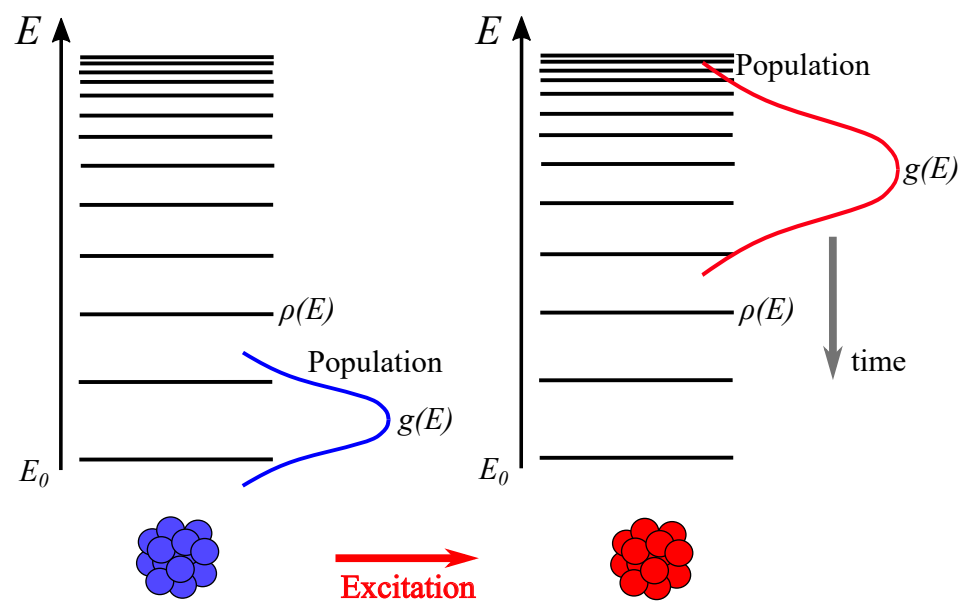

Figure 6: Schematic representation of the processes of excitation and cooling of an ensemble of clusters and their population over vibrational states. The figure does not include excited electronic

states.

The total decay rate can then be expressed as

$$
R(t)=\int_{0}^{\infty} g(E, 0) \mathrm{e}^{-k(E) t} k(E) \mathrm{d} E
$$

Clearly, this reduces to a single exponential decay if the excitation energy is found in a very narrow distribution centered at $E_{0}$, making $g(E, 0) \stackrel{\propto}{\approx} \delta\left(E-E_{0}\right)$;

$$
R(t)=\int_{0}^{\infty} \delta\left(E-E_{0}\right) \mathrm{e}^{-k(E) t} k(E) \mathrm{d} E=\mathrm{e}^{-k\left(E_{0}\right) t} k\left(E_{0}\right) .
$$

Matters change for broad energy distributions. These will arise from a number of factors, such as laser beam profiles, Poisson photon absorption statistics, stochastic energy loss carried away by electrons or atoms during ionization and initial cluster decays, etc. In experiments employing strong laser pulses to excite and ionize clusters in multiphoton processes with photon energies below threshold, a significant width on the internal energy distribution is virtually unavoidable. For the commonly used Gaussian laser beams also the fluence profile across the beam will cause a significant broadening of the energy distribution [57]. For these commonly encountered situations, the calculation of the integral in Eq. (7) can be simplified by noting that the factor $\mathrm{e}^{-k(E) t} k(E)$ in the integrand in Eq. (9) is a strongly peaked function of energy. This is exemplified for $\mathrm{Au}_{9}^{+}$in Fig. 7, where the fragmentation rate $k(E)$ is compared with $\mathrm{e}^{-k(E) t} k(E)$. 


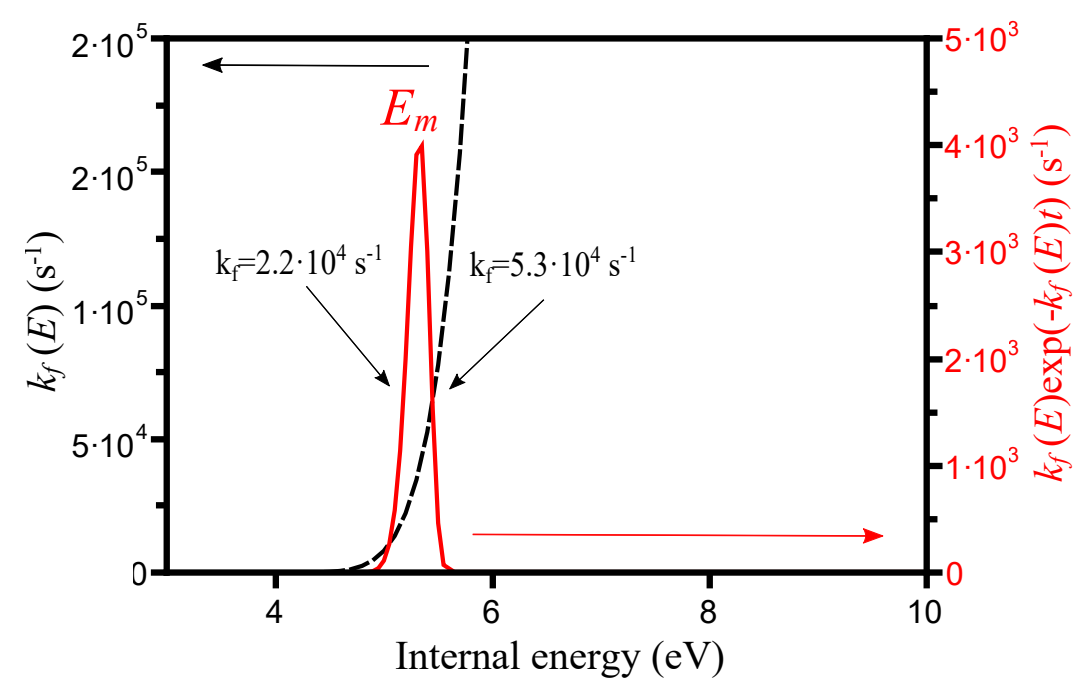

Figure 7: Plots of the fragmentation rate constant, $k_{f}(E)$ (black dashed line), and the function $k_{f}(E) \exp \left(-k_{f}(E) \cdot t\right.$ ) (red line) for the $\mathrm{Au}_{9}^{+}$cluster as a function of excitation energy. For the plots, level densities were approximated by the high-energy limit of harmonic oscillators [46]. The measured dissociation energy of [42], and the frequency factor calculated in [58] were used, together with a time $t=90 \mu \mathrm{s} . E_{m}$ represents the energy at which $k_{f}(E) \exp \left(-k_{f}(E) t\right)$ peaks. For reference, the two rate constants corresponding to the half-maximum values of the function $k_{f}(E) \exp \left(-k_{f}(E) t\right)$ are indicated. The ratio of the two is close to $\exp (1)$, which holds generally for pairs of such half-width values. The curve $k_{f}(E) \exp \left(-k_{f}(E) t\right)$ has the peak value $\exp (-1) / t$, as expected on general grounds [46].

The narrow energy interval over which decays occur at a specific time means that the integral in Eq. (9) can be approximated by evaluating $g(E)$ at the energy, $E_{m}$, at which $\mathrm{e}^{-k(E) t} k(E)$ peaks. Hence,

$$
R(t) \cong g\left(E_{m}\right) \int_{0}^{\infty} \mathrm{e}^{-k(E) t} k(E) \mathrm{d} E=g\left(E_{m}\right) \int_{0}^{\infty} \frac{k}{k^{\prime}} \mathrm{e}^{-k t} \mathrm{~d} k
$$

where the variable change $\mathrm{d} E=(\mathrm{d} E / \mathrm{d} k) \mathrm{d} k \equiv k^{\prime-1} \mathrm{~d} k$ was used. The exponential is the strongest varying factor in the integrand. The factor $k / k^{\prime}$ is slowly varying in comparison and can therefore be evaluated at $E_{m}$. This gives

$$
R(t) \cong g\left(E_{m}\right) \frac{k\left(E_{m}\right)}{k^{\prime}\left(E_{m}\right)} \int_{0}^{\infty} \mathrm{e}^{-k t} \mathrm{~d} k=g\left(E_{m}\right) \frac{k\left(E_{m}\right)}{k^{\prime}\left(E_{m}\right)} \frac{1}{t} .
$$

It should be clear from the derivation and the close similarity of Eq. (3) and Eq. (4) that this $1 / t$ decay law holds for both fragmentation and electron emission, and only 
relies on the rate constant of the dominant process being a rapidly increasing function of the excitation energy, in addition to the requirement of a broad energy distribution. It is important to note, though, that the $1 / t$ decay will only be observed when the detected channel is the dominant channel, whether fragmentation or ionization. Otherwise another rate constant will appear in the argument of the exponential describing the decay. See [59] for an example of this case.

Another important aspect of a power law decay is less obvious from the derivation; viz. the fact that the activation energy of the observed channel will set the energy scale of the clusters with that decay. High activation energies mean high effective temperatures and therefore easier thermal access to excited electronic states.

The $1 / t$ decay rates have been observed in several experiments on highly excited clusters studied in storage rings, linear ion traps and conventional time-of-flight mass spectrometer setups $[43,56,60-65]$. Two examples are shown in Fig. 8. Panel (a) shows the neutralization yield of $\mathrm{Ag}_{5}^{-}$clusters produced in a sputter source and measured as a function of time in the electrostatic storage ring ELISA [56]. An electrostatic storage ring is a device where ions can be stored for extended periods of time, moving in welldefined trajectories with high kinetic energies. The long storage time combined with the high detection efficiency of neutral decay products in these devices allow efficient measurements of decay rates over extended periods of time. As observed in the figure, the neutral yield follows a $1 / t$ decay closely over two and a half order of magnitude in time. Panel (b) of Fig. 8 shows the metastable fragmentation of $\mathrm{Au}_{18}^{+}$to $\mathrm{Au}_{17}^{+}$, with data measured in a reflectron time-of-flight mass spectrometer by the cluster group at KU Leuven [43]. The line in the figure shows a fit with the integrated form of a $1 / t$ decay rate.

The use of reflectron time-of-flight mass spectrometers is common, and their application to radiation studies is convenient. The procedure used to generate the data in Fig. 8 will therefore be given in some detail. A schematic view of the setup is shown in Fig. 9. A cluster beam is produced upstream and guided to the position shown, where it is exposed to a strong laser pulse which excite the clusters and cause extensive fragmentation. The zero of time is defined as the absorption of the photons from the ns duration laser pulse. After acceleration of the charged clusters in a pulsed field they travel some field-free distance until they reach the reflectron, which acts as an electrostatic mirror. After turning in the reflectron, they are directed toward the detector which records their arrival time.

By tuning the potentials in the reflectron, the device allows measurements of two different kinds of fragmentation products; those that occur before the initial acceleration 
(a)

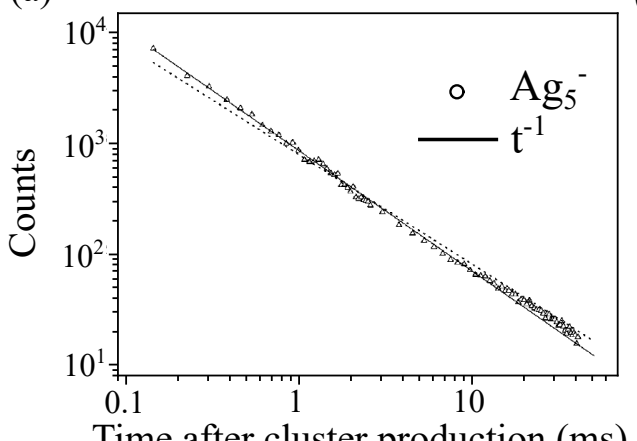

(b)

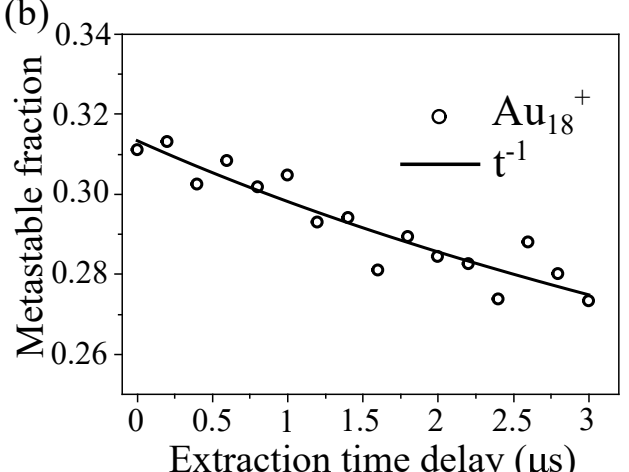

Figure 8: (a) Yield of neutrals detected in a storage ring after injection of anionic $\mathrm{Ag}_{5}^{-}$clusters decaying by electron emission. (b) Metastable fraction of cationic $\mathrm{Au}_{18}^{+}$ clusters, decaying by neutral monomer emission, as a function of delay time in the extraction. The abscissa is exclusive the zero delay acceleration time which is a few $\mu$ s. In both panels a power law decay is compared to the experimental data, in panel (a) directly, and in panel (b) in the appropriate integrated form, given in Eq. (13). Frame (a) reprinted with permission from [56]. Copyright (2001) by the American Physical Society. Frame (b) reprinted with permission from [43]. Copyright (2017) by the American Physical Society.

is over, lasting on the order of a few $\mu s$ and that do not fragment further before entry into the reflectron (prompt product), and those that fragment between the acceleration stage and the entry into the reflectron (metastable component). The latter type will generally be present in measurable quantities for $1 / t$ decays. Obviously the distinction between prompt and metastable fragmentation depends on known instrumental parameters such as lengths and voltages.

The mass spectrometer can be used to measure the metastable decay because a prompt fragment is accelerated and reflected with the same mass ( $m$ in Fig. 9), whereas a cluster that decays in free flight to size $m^{\prime}$ after the initial acceleration will lose translational kinetic energy in proportion to the lost mass, $m-m^{\prime}$. By a suitable tuning of the reflectron potentials, the arrival time at the detector of this fragment can be displaced from the arrival times of both the clusters accelerated with the masses $m$ and $m^{\prime}$. This shift allows the identification of clusters that have decayed metastably and provides a quantitative measure of the yield of this process.

An example of a mass spectrum measured with this procedure is shown in Fig. 10 for photo-fragmented $\mathrm{Au}_{N}^{+}$clusters [43]. The metastable fragments can be recognized as additional peaks with an apparent number of atoms which is not integer. With only a 


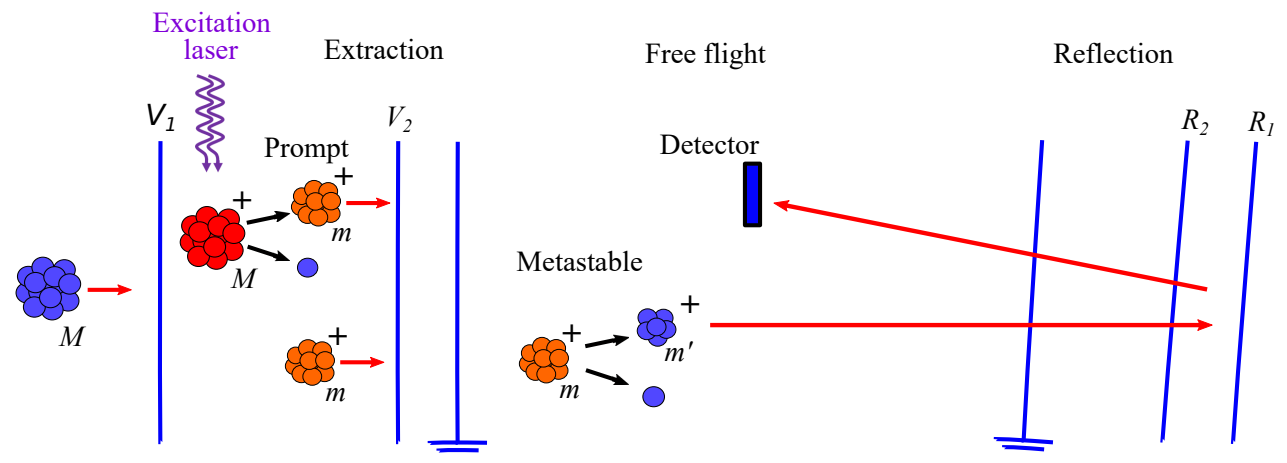

Figure 9: Scheme of a reflectron time-of-flight mass spectrometer, showing the extraction and reflection stages of the system. Laser light excites a cluster beam within a two-stage extraction stage, where they are accelerated by a potential given by $V_{1}$ and $V_{2}$ and their starting position. The clusters then fly freely until they reach the reflectron, which consists of a two-stage static electric field, where they are decelerated and reflected. Finally, the clusters hit the detector, which records their arrival time. Prompt fragmentation takes place within the extraction stage, metastable fragmentation during free flight.

single mass spectrum, it is therefore possible to identify the fragmentation channels of the photo-excited clusters and the amount of metastable fragmentation through that channel. Fig. 10 (b) shows the metastable fractions, defined as the normalized metastable fragment intensities for both the active channels of monomer and dimer evaporation, extracted from the mass spectrum of panel (a) of Fig. 10. The strong oscillation between monomer and dimer evaporation as the preferred fragmentation channel in the $N=6-16$ size range is a consequence of the odd-even stability pattern of $\mathrm{Au}_{N}^{+}$clusters already seen by Katakuse [66] in mass abundance spectra and later quantified in Ref. [42].

In order to obtain curves of the type shown in frame (b) of Fig. 8, it is necessary to vary the extraction time in the initial acceleration in the time-of-flight mass spectrometer. This is accomplished by introducing a delay between the laser pulse and the switching on of the electric field. A detailed description of this procedure can be found in Ref. [67].

The reflectron time-of-flight experiments thus measure the integrated decay rate, which after normalization is known as the metastable fraction, between the two instrumentally defined times that are given by the mass selection time in the initial acceleration, $t_{1}$, and the time of entry into the reflectron, $t_{2}$. In the absence of radiation the metastable fraction is

$$
M \propto \int_{t_{1}}^{t_{2}} R(t) \mathrm{d} t \propto \ln \left(t_{2} / t_{1}\right) .
$$

Experiments where the start and end of the time window are changed, thus changing the 

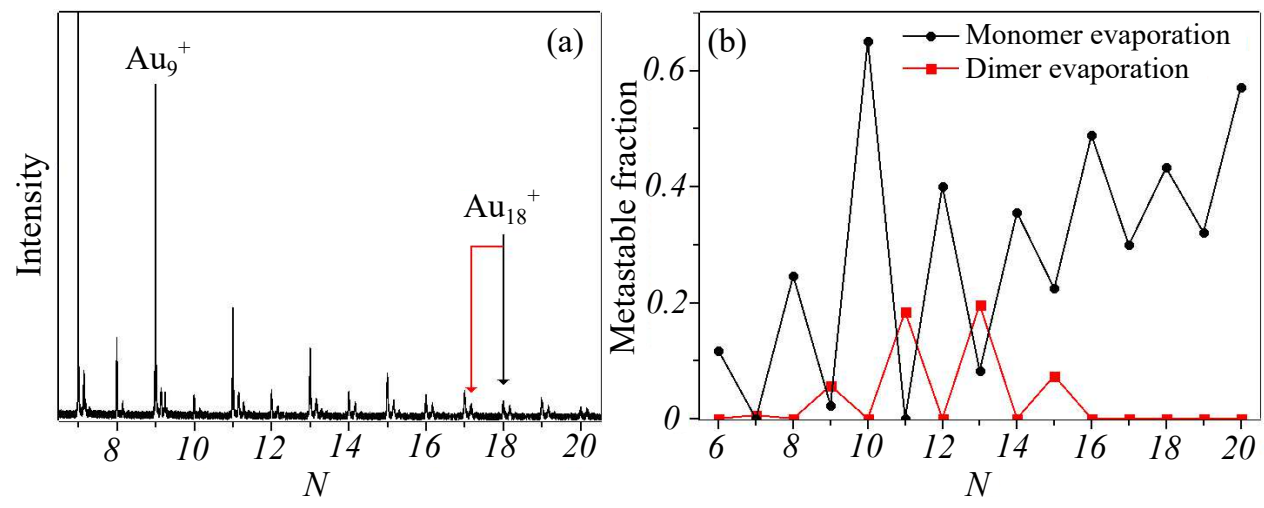

Figure 10: (a) Part of a mass spectrum of photofragmented $\mathrm{Au}_{N}^{+}$clusters that are produced by laser ablation and inert gas condensation, followed by excitation with an intense pulse of $\lambda=355 \mathrm{~nm}$ laser light. The fragmentation channel $\mathrm{Au}_{18}^{+} \rightarrow \mathrm{Au}_{17}^{+}+$ $\mathrm{Au}$ is indicated by arrows. (b) Metastable decay fraction versus cluster size monomer (circles) and dimer (squares) evaporation, extracted from the mass spectrum of panel (a). Reprinted with permission from [43]. Copyright (2017) by the American Physical Society.

ratio $t_{2} / t_{1}$ in an experimentally controlled fashion, allow a comparison with the expected power law decay. Panel (b) in Fig. 8 shows the amount of metastable decay as a function of such a variation of the onset of the time window.

\subsection{Radiative quenching of unimolecular decay}

The presence of radiation will modify the power law decay rate. The effect is particularly simple to describe for the case where the energy of the photon is sufficiently high for a single photon emission to quench any future fragmentation [67]. The populations of excited states then decreases as

$$
\frac{\mathrm{d} g(E, t)}{\mathrm{d} t}=-\left(k_{p}(E)+k(E)\right) g(E, t),
$$

where $k_{p}(E)$ is the rate constant for photon emission.

As illustrated in Fig. 3 for $\mathrm{Au}_{9}^{+}, k_{p}$ has a much weaker energy-dependence than the $k$ rate constant for both dissociation and electron emission. As discussed in section 2, this generally holds. We can therefore in the first approximation treat $k_{p}$ as a constant and express $R(t)$ as

$$
R(t)=\int_{0}^{\infty} g(E) \mathrm{e}^{-\left(k_{p}+k(E)\right) t} k(E) d E \cong g\left(E_{m}\right) \mathrm{e}^{-k_{p} t} \int_{0}^{\infty} \mathrm{e}^{-k(E) t} k(E) d E .
$$


The integral is identical to the one calculated previously for the non-radiative case, and the unimolecular rate therefore becomes

$$
R(t) \cong g\left(E_{m}\right) \frac{k\left(E_{m}\right)}{k^{\prime}\left(E_{m}\right)} \frac{\mathrm{e}^{-k_{p} t}}{t} .
$$

As $k_{p}$ is the rate constant for an activated process, it is in principle energy dependent and can therefore not rigorously be taken constant. A calculation of the leading order correction caused by the energy dependence of $k_{p}$ corrects fitted values of $k_{p}$ to the slightly different value $k_{p}\left(1-h \nu / E_{a}\right)$, where $h \nu$ is the energy of the photon emitting state and $E_{a}$ the activation energy of the observed, competing decay channel [68].

It was implicitly assumed above that photons are emitted from a single level. A more general expression will include contributions from several levels. This is not an unrealistic situation, see for example $[69,70]$. Apart from an increase of the radiated power, this only affects the decay curves marginally due to the relatively constant value of radiative time constants vs. energy. The main effect of two or more emitting states will be to replace $k_{p}$ by a sum of the radiative rate constants involved. In summary, for the cases discussed so far, that correspond to large photon energies (for a precise difinition see section 4), the radiative modifications to the integrated decay rate in Eq. (13) is given by the exponential integral [71], Ei, as

$$
M \propto \int_{t_{1}}^{t_{2}} \frac{\mathrm{e}^{-k_{p} t}}{t} \mathrm{~d} t=\operatorname{Ei}\left(k_{p} t_{1}\right)-\operatorname{Ei}\left(k_{p} t_{2}\right)
$$

For emission of photons with small energies, the emission rate and the corresponding integrated metastable rates take a slightly different form. In this limit, where the cooling can be considered continuous, the decay rate is obtained by including a time dependence into the rate constant $k$, in addition to its energy dependence. It is found by expanding the logarithm of $k(E, t)$ in time yielding, to leading order [19],

$$
k(E, t)=k(E, 0) \mathrm{e}^{-\mathrm{w} t}
$$

with

$$
\mathrm{w} \equiv-\frac{\mathrm{d} \ln (k)}{\mathrm{d} t}
$$

The expression corresponds to a variation of the rate constant with energy that can be approximated with a first order exponential function and a constant emitted power. Although logarithms of quantities with physical units are undefined, derivatives of such logarithms are well defined and independent of the unit system used. See p. 15 of [46] for a short discussion of this. With this time dependence of the rate constant, the analogue of the large photon energy expression in Eq. (7) becomes

$$
R(t)=\int_{0}^{\infty} g(E, 0) \mathrm{e}^{-k(E, 0) \int_{0}^{t} \mathrm{e}^{-\mathrm{w} t} \mathrm{~d} t} k(E, 0) \mathrm{e}^{-\mathrm{w} t} \mathrm{~d} E
$$


where now the time dependence is on the rate constant as opposed to the population in the large photon energy case. Performing the integral in the exponential gives

$$
R(t)=\int_{0}^{\infty} g(E, 0) \exp \left(-\frac{k(E, 0)}{\mathrm{w}}\left(1-\mathrm{e}^{-\mathrm{w} t}\right)\right) k(E, 0) \mathrm{e}^{-\mathrm{w} t} \mathrm{~d} E .
$$

Defining a fictitious time, $\tau$, as

$$
\tau \equiv \frac{1-\mathrm{e}^{-\mathrm{w} t}}{\mathrm{w}}
$$

the decay rate can be written as

$$
R(t)=\mathrm{e}^{-\mathrm{w} t} \int_{0}^{\infty} g(E, 0) \mathrm{e}^{-k(E, 0) \tau} k(E, 0) \mathrm{d} E .
$$

Apart from the exponential prefactor, this is identical to the non-radiative case if $\tau$ is substituted for $t$. Calculating the integral with the same approximations as for the nonradiative case and reinserting the definition of $\tau$ then gives the decay rate

$$
R(t) \cong g\left(E_{m}^{\prime}\right) \frac{k\left(E_{m}^{\prime}\right)}{k^{\prime}\left(E_{m}^{\prime}\right)} \frac{\mathrm{w}}{\mathrm{e}^{\mathrm{w} t}-1}
$$

where $E_{m}^{\prime}$ is the energy at which $\exp \left(\frac{-k(E, 0)}{\mathrm{w}}\left(1-\mathrm{e}^{-\mathrm{w} t}\right)\right) k(E, 0) \mathrm{e}^{-\mathrm{w} t}$ peaks. This provides the suppression of the power law in the small energy photon limit.

The low photon energy analogue of Eq. (17) for the metastable decay fraction is given by the integral of Eq. (24) between times $t_{1}$ and $t_{2}$. If the radiation is a small perturbation to the power law decay, the integral can be expressed as $[22,72]$

$$
M \propto \ln \left(t_{2} / t_{1}\right)-\frac{1}{2} \mathrm{w}\left(t_{2}-t_{1}\right)
$$

As the last term is often constant in the measurements, $M$ plotted vs. $\ln \left(t_{2} / t_{1}\right)$ will give a straight line with a negative intercept if radiation is present. The ratio of this intercept to the slope of the line will cancel the constant of proportionality and give $\mathrm{w}$ in terms of the two instrumental times. A similar expansion holds for the large photon energy case, with $\mathrm{w}$ replaced by $k_{p}$ and the factor $1 / 2$ by 1 .

The two parameters $k_{p}$ and w characterizing the high and low photon energy regimes are both coefficients quantifying the presence of radiation and both have dimensions of inverse time. They also give rise to very similar temporal dependencies. With identical numerical values of $k_{p}$ and $\mathrm{w}$, the main difference is that the former decreases a factor of 1.3 faster than the latter [73]. However, for the interpretation of experimental data it should be kept in mind that they have different physical meaning. From the derivations it is clear that for the large photon energy, the exponential decrease of $R(t)$ with time 
has the same origin as the standard exponential decay, viz. a exponential decrease of the population of the decaying states. The exponential modification of the power law decay for the small photon expression in Eq. (24), on the other hand, is derived from an exponential decrease of the rate constants, $k(E, t)$.

Both measurements of decay rates and integrated decay rates have been used to identify the presence of radiation as a cooling channel for a number of systems, mainly clusters. Fig. 11 gives three examples. Panel (a) shows the electron emission decay rates of anionic
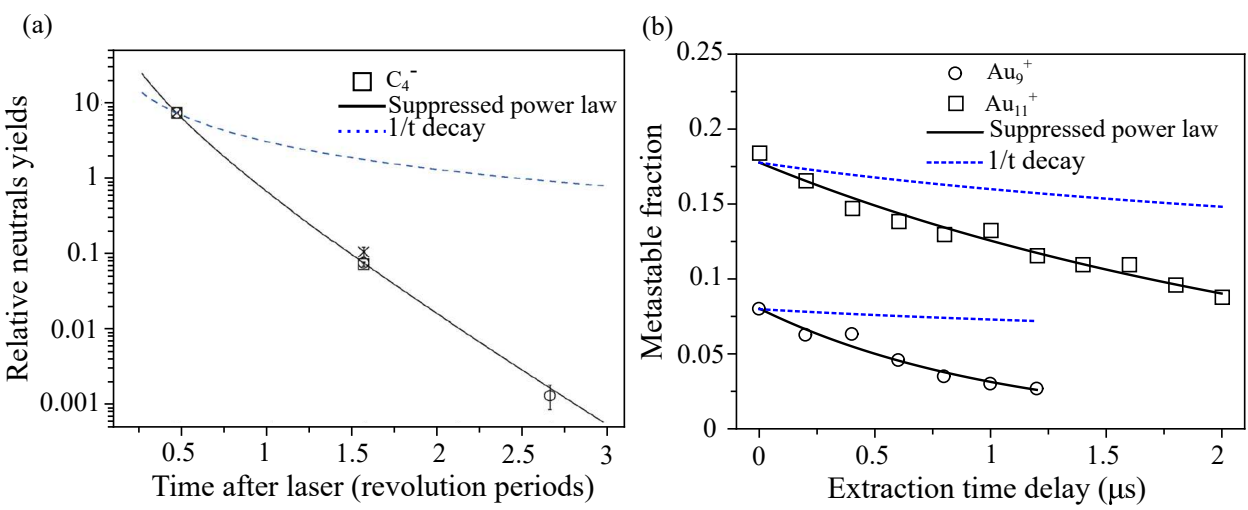

Figure 11: (a) Yield of neutrals detected turn by turn in a storage ring after injection of anionic $\mathrm{C}_{4}^{-}$clusters. The different (hardly discernible) symbols represent different wavelengths and firing times of the excitation laser (Ref. [69] - Reproduced by permission of the PCCP Owner Societies). (b) Metastable fraction of the cationic $\mathrm{Au}_{9}^{+}$and $\mathrm{Au}_{11}^{+}$clusters as a function of delayed time of extraction. Reprinted with permission from [43]. Copyright (2017) by the American Physical Society. In both panels a power law decay (dashed line) is also plotted, showing that the decreases of the decay rate are much faster than $1 / t$ in all three cases. The continuous lines represent a fit with an exponentially quenched power law. Note that quantities plotted are different in the two frames, one is the decay rate, the other the integrated yield. The plot in frame (b) involves instrumental times and the dotted, non-radiative curve is therefore not $1 / t$.

$\mathrm{C}_{4}^{-}$clusters after laser excitation of the ions in an electrostatic storage ring [69]. The measured points are the decay rates at each turn of the ions in the ring. Different ion storage times (waiting times before laser excitation) and different photon energies were examined. They are shown in the figure, normalized to the first point. The decay is much faster than a power law, showing the presence of radiative cooling competing with the measured signal of electron emission. Different excitation parameters give identical decays, demonstrating that the decay is determined by internal properties of the clusters, and does not result from source conditions. It also demonstrates unambiguously that the 
quasi-exponential decay is not the result of a single exponential decay that would arise from a delta-function excitation energy distribution of a thermally activated process. As is well known, such unimolecular process would show a strong dependence on the energy of the absorbed photon. Another case is shown in Fig. 11b, for the cationic $\mathrm{Au}_{9}^{+}$and $\mathrm{Au}_{11}^{+}$clusters. The channel is fragmentation, measured as the metastable decay fraction in the reflectron time-of-flight mass spectrometer described above.

The radiative rate constants measured for these three clusters are all orders of magnitude higher than time constants associated with any vibrational transitions, pointing clearly to an origin of the photons in thermally populated electronic excitations. For the $\mathrm{C}_{4}^{-}$case, the excited states are known experimentally from spectroscopic studies and the measured rates reproduce these facts very well [69]. For the gold clusters, much less is known about the emitting states and only limits can be placed on the energies of the photon emitting states [43].

As the case of the gold clusters shows, the quenched power law decay provides a convenient tool for observing the radiative decay, but it will not give all information of the radiative channel without some additional input. In particular, it is not possible to determine absolute energy emission rates from the quenching of the power law decay alone. A measurement of energy loss per time unit can not be derived from measurements of time constants alone.

A possibility to measure emitted power is, however, available. The method is based on the fact that power law decays, including the quenched variety, have a zero of time. This sets them apart from exponential decays which have a characteristic time but not a zero of time. The procedure consists of reheating the clusters at some specific time with a single photon absorption. The photo-excited clusters will decay with an enhanced rate compared to the spontaneous contribution. Shifting this observed enhanced decay backwards in time to a point where the (logarithmic) slope of it is identical to the corresponding value for the spontaneous decay gives directly the time interval over which the clusters have emitted one photon energy. In this procedure, the energy scale, otherwise absent in the problem, is provided by the photon energy, and the time scale by the reheating time shift. Fig. 12 illustrates the procedure for $\mathrm{C}_{60}^{-}$measured in a storage ring.

The cooling measured in these experiments is in general the sum of radiative cooling and depletion by the spontaneous decay intrinsic to the powerlaw decay, radiatively quenched or not. The absolute cooling curve of such a study on $\mathrm{C}_{60}^{-}[74]$ is shown in Fig. 13. The figure includes the radiative cooling rate determined from the spontaneous decays in [20] for a single time (5 ms) with the value $100 \mathrm{eV} / \mathrm{s}$, in good agreement with the results here. The method is applicable also for the curves influenced by radiative cooling alone, 


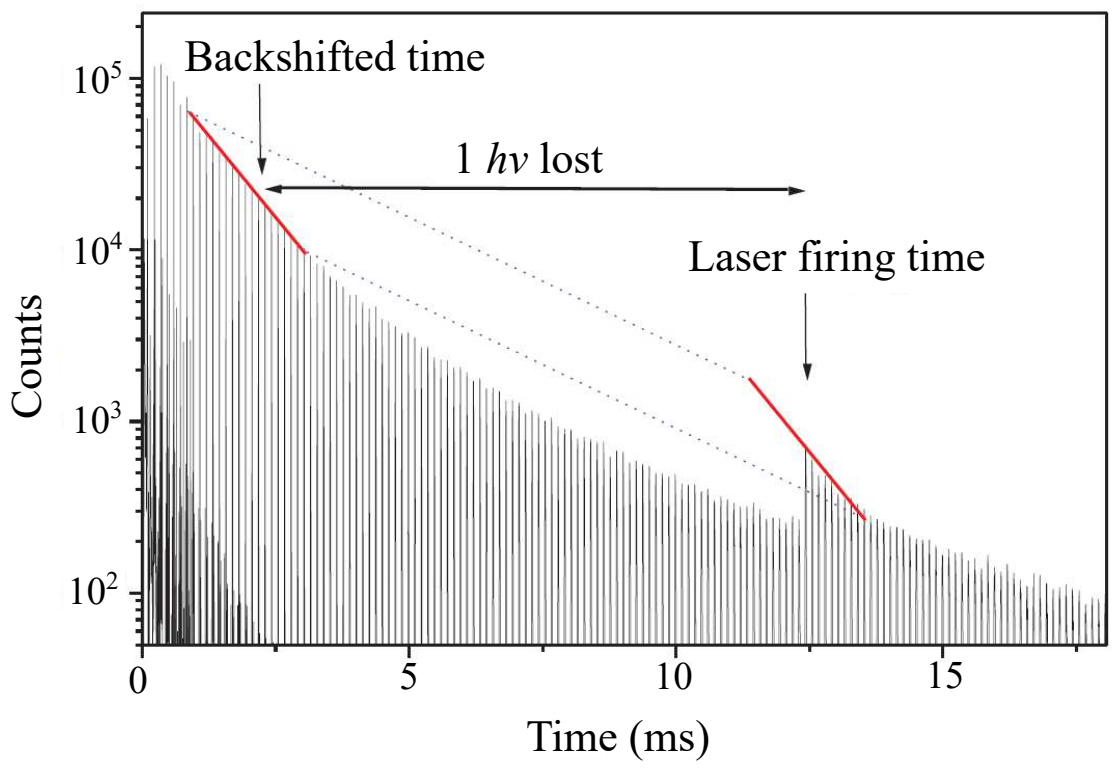

Figure 12: The procedure used to find the time during which the stored $\mathrm{C}_{60}^{-}$loses one photon energy. The excitation photon energy was $2.0 \mathrm{eV}$ in this case. The data were published in [74].

with no unimolecular decay present. The method requires that the measured decay rates are sufficiently different from exponential decays.

\section{The boundary between large and small photon en- ergies}

An exponential suppression was derived in Eq. (16) for the quenching effect of radiation on the power law decay. That analysis is only valid if the emission of a single photon is sufficient to quench the unimolecular decay. In the other limit of small photon energies, the non-radiative power law is quenched by emission of low energy photons in what is effectively a continuous process with a decay profile given by Eq. (24). In this section we establish the boundary between the two regimes in terms of the photon energy and other relevant parameters.

The boundary separating the two types of quenching processes is defined by the magnitude of the change of the unimolecular rate constant upon emission of one photon. A large photon energy is defined as one that will quench any further unimolecular decay. The small photon energy, or continuous cooling limit, corresponds equivalently to a small 


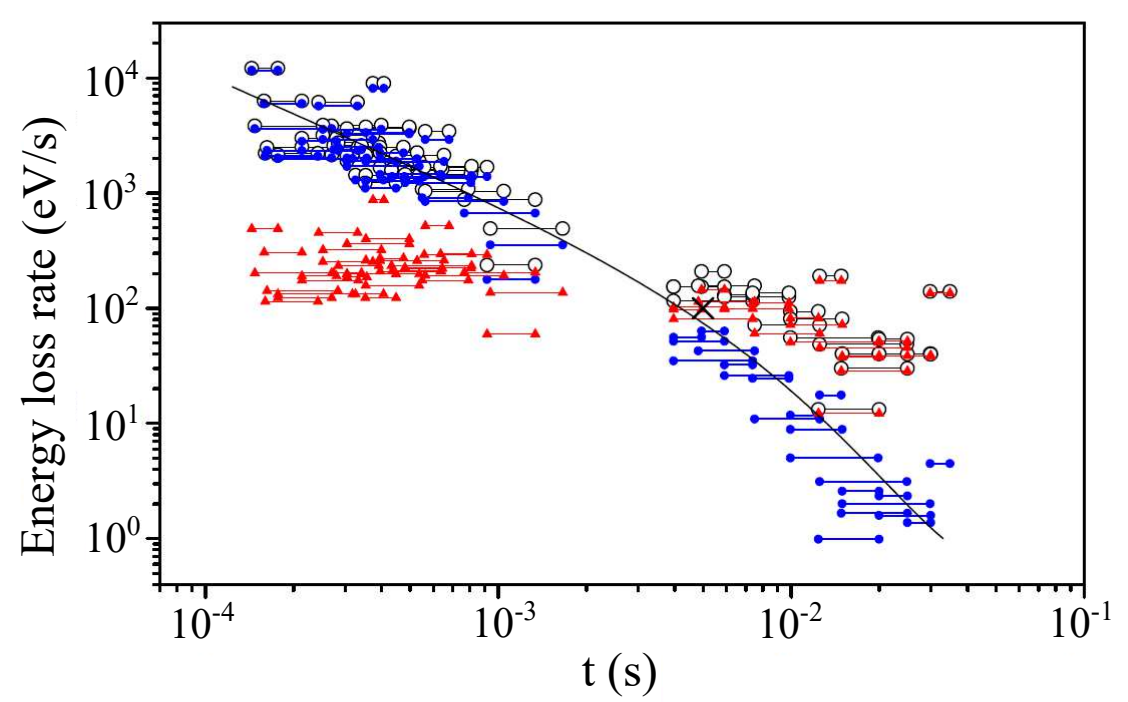

Figure 13: The measured absolute energy loss from the high energy edge of excited $\mathrm{C}_{60}^{-}$ as a function of time. Cooling rates for different laser firing times and photon energies are subtracted to get the points shown. Black open circles give the total cooling rate, blue filled circles the cooling by depletion, and red triangles the radiative cooling. The line is the depletion cooling rate derived from the observed spontaneous decay rate. It should be compared with the blue filled circles. The point from [20] is given with a black cross. The point should be compared with the curve given by the red triangles.

relative change of the unimolecular rate constant after photon emission, i.e.

$$
\frac{\mathrm{d} \ln (k)}{\mathrm{d} E} h \nu \lesssim 1,
$$

where $h \nu$ is the energy of the emitted photons and $k$ is the relevant unimolecular rate constant (for fragmentation or electron emission). The photon energy corresponding to the equality sign is roughly equal to the width of the peak in Fig. 7.

An important observation is that in the derivation of expressions for the decay rates, no assumptions were made of the physical mechanism responsible for the photon emission process. A consequence of this is that the distinction between the high and low photon energy regimes is not the result of the mechanism underlying the photon emission process, in particular whether it is emission from vibrationally or electronically excited states. Other parameters decide this question, most importantly the heat capacity $C_{v}$ and the dissociation energy $D$ (or the electron binding energy). Considering the decays of the hottest clusters in a beam, that are the ones that give the measured signals, we can separate the parameter space in three regions, as shown in Fig.14. 


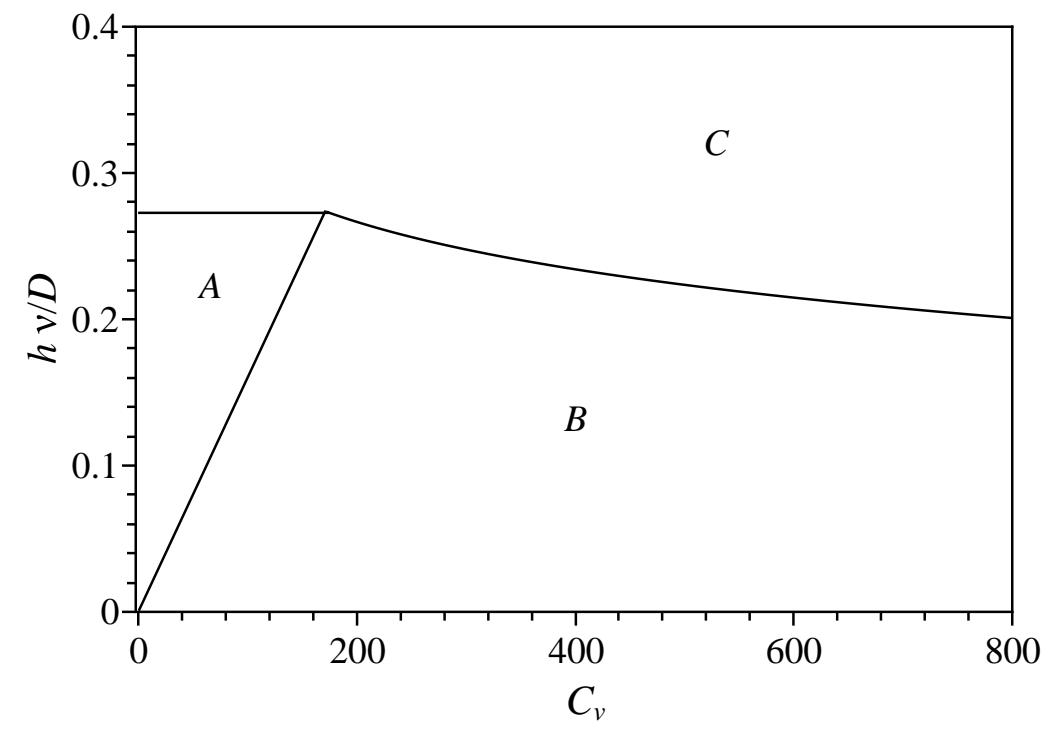

Figure 14: The different regions for photon emission vs. particle heat capacity. Region $\mathbf{A}$ is the parameter space where a single photon emission quenches fragmentation. In region $\mathbf{B}$ the quenching is by continuous cooling, and in region $\mathbf{C}$ fragmentation dominates and radiative cooling can be neglected. The derivations of the boundaries of the three regions are given in the main text. See also [73]. The parameters used in the figure are $\omega=10^{15} \mathrm{~s}^{-1}, t=100 \mu \mathrm{s}, A=10^{7} \mathrm{~s}^{-1}$.

In region $\mathbf{A}$ fragmentation (or electron emission, we will use the term fragmentation for simplicity) is quenched by the emission of a single photon. This region has a boundary to $\mathbf{B}$ by the complement to the inequality in Eq. (26). With an Arrhenius expression for the unimolecular rate constant, which includes the frequency factor $\omega$, the boundary is, with the (average) emitted photon energy $h \nu$, given by Eq. (26) with an equality sign

$$
\frac{h \nu}{D}=\frac{C_{v}}{\ln (\omega t)^{2}},
$$

where $D$ is the unimolecular activation energy, $C_{v}$ is the heat capacity in units of $k_{B}$, and the time in the denominator may be replaced by $1 / k_{p}$. The boundary between $\mathbf{A}$ and $\mathbf{C}$ is defined by the requirement that the photon emission is competitive with the unimolecular reaction. With Eq. (1), the Arrhenius unimolecular rate constant, and the estimate $T=D / \ln (\omega t)$ from the Arrhenius expression, the boundary is located at

$$
\frac{h \nu}{D}=\frac{1}{\ln (\omega t)} \ln (1+A t),
$$

where $A$ is the Einstein coefficient for spontaneous photon emission from the state considered. 
In region $\mathbf{B}$ fragmentation is quenched by continuous cooling. The boundary between $\mathbf{B}$ and $\mathbf{C}$ is defined by requiring that

$$
\frac{k_{a}}{\mathrm{w}} \lesssim 1
$$

This translates into the condition

$$
\frac{1}{A t} \frac{D}{h \nu} \frac{C_{v}}{(\ln (\omega t))^{2}}\left((\omega t)^{\frac{h \nu}{D}}-1\right) \lesssim 1
$$

This must be solved numerically for $h \nu / D$.

Finally, in region $\mathbf{C}$ fragmentation dominates the decay and radiation can be neglected. Fig. 14 shows these regions calculated with the parameters given in the figure caption.

Although the precise borders of the regions in Fig. 14 require a calculation with specified parameters, we can understand the features qualitatively. For small values of $h \nu$ relative to $D$, emission of a single photon is insufficient to suppress fragmentation, which places us in region B. For larger photon energies and/or smaller heat capacities, emission of a single photon can be enough to quench any further fragmentation, corresponding to a case located in region $\mathbf{A}$. The crossover from region $\mathbf{A}$ to $\mathbf{C}$, by increasing the value of $h \nu$ relative to $D$, is a consequence of the fact that although fragmentation and photon emission are both activated processes, the frequency factor associated with fragmentation is much larger than that of radiation. The same argument applies to the cross-over from region $\mathbf{B}$ to region $\mathbf{C}$ when the photon energy increases and the photon emission activation energy becomes too high for this channel to be competitive.

Both the borders between $\mathbf{A}$ and $\mathbf{C}$ and between $\mathbf{B}$ and $\mathbf{C}$ will move up with time. Photon energies once too high for the radiative channel to compete with the unimolecular decay will at a later time move into the radiative regime. No cluster will cross between the regions $\mathbf{A}$ and $\mathbf{B}$, however.

In summary, decay rates will take the form

$$
R(t) \propto \frac{\mathrm{e}^{-k_{p} t}}{t}
$$

for large photon energies (region A in Fig. 14), and

$$
R(t) \propto \frac{1}{\mathrm{e}^{\mathrm{w} t-1}},
$$

for small photon energies (region B in Fig. 14). 


\section{The effect of radiation on abundances}

Fragmentation, both laser induced and spontaneous, are frequently used to identify particularly stable clusters. Rate constants depend strongly on the evaporative activation energies, which for clusters in most, if not all, cases are equal to separation energies, i.e. the differences of total ground state energies of the decaying cluster and the decay products. If a distribution of hot clusters spanning a range of different sizes is measured, the intensities of the individual cluster sizes will reflect this measure of their stability.

Seminal experiments that revealed the non-monotonous size dependence of cluster properties, the electronic shell structure of sodium clusters in 1984 by Knight et al. [2] and the atomic packing shell structure of xenon clusters in 1981 by O. Echt et al. [1], both used ensembles of hot clusters. Irrespective of the different origin of these two types of shell structure, the measured abundances reflect in both cases relative cluster stabilities. A third case of a highly nontrivial size dependence are the fullerenes, of which $\mathrm{C}_{60}$ is the best known representative, which recently attracted renewed interest after the discovery of its presence in space $[75,76]$. Although the precise production mechanism of these all-carbon molecules is still debated, there is ample evidence to show that the molecules

emit thermal photons in competition with both electron emission and unimolecular loss of $\mathrm{C}_{2}$. This will stabilize the molecules that radiate with particularly high rates and enhance their abundances, both in quasi-equilibrium and for free molecules. The mechanism will obviously be in effect for any other homologous series of molecules or clusters that radiate thermally.

The possibility to identify and quantify the stability of clusters by their hot ensemble abundances is so useful that it is also applied experimentally to cold sources, for example when clusters are produced with laser ablation [77], sublimation [78], or magnetron sputtering in combination with inert gas cooling [79]. The clusters must then be excited prior to detection to develop the abundance contrasts. This may be achieved by photoexcitation or by collisional heating. Irrespective of the mode of production, the measured abundances will be influenced by any radiative cooling present. It is therefore of interest to know how this will affect the abundance-stability relation.

\subsection{Non-radiative abundances}

Abundances of hot clusters in a molecular beam do not map stabilities directly. Fundamentally, this is due to the absence of an external heat bath to which the clusters can thermalize. This endows hot cluster molecular beams with special features that influence the relation between stability and abundances. In the absence of radiation, this 
relation is well understood [80], and has been used to extract dissociation energies from experimental data [81-83]. The situation where radiative cooling is the only de-excitation channel present has also been analyzed [84], but will not be discussed in detail here. In this section, we describe the intermediate case between these two situations, for which both evaporative and radiative cooling are present and compete.

The non-radiative relation is given by [80]

$$
I_{N} \propto \frac{1}{2}\left(D_{N}^{\prime}+D_{N+1}^{\prime}\right)+\frac{C_{v, N}}{G}\left(D_{N}^{\prime}-D_{N+1}^{\prime}\right)
$$

where $I_{N}$ is the intensity in mass spectra of cluster size $N$, which has heat capacity $C_{v, N}$ (in units of Boltzmann's constant). The dissociation energies are $D_{N}$ and the prime indicates a correcting term on the bare values [46], which is $3 \hbar \bar{\omega} / 2$ for the harmonic approximation and loss of one atom. The dimensionless quantity $G_{N} \equiv \ln \left(\omega_{N} t_{N}\right) \simeq G$, with $\omega_{N}$ the frequency factor of the rate of fragmentation, is known as the Gspann parameter [85, 86] and has a value of typically 25-30. It is fairly independent of size, as indicated by the use of a universal value for all cluster sizes. $t_{N}$ is the time between creation of the cluster and the measurement of the abundances in the experimental setup. The constant of proportionality in the expression is size dependent, but the dependence is a smooth function of size (see also [46]).

An example of the use of Eq. (33) is given in Fig. 15, applied to laser excited $\mathrm{Pt}_{N}^{+}$ clusters in a time-of-flight mass spectrometer [65]. In panel (a) the metastable fractions versus time delay of extraction are shown for $\mathrm{Pt}_{3}^{+}$and $\mathrm{Pt}_{8}^{+}$. In both cases, the decays follow a power law, indicating the absence of radiative cooling on the time scale of the experiment. This also holds for all other platinum clusters measured in this experiment. We can therefore apply the non-radiative relation Eq. (33). In this example, instead of inverting Eq. (33) to obtain experimentally observed dissociation energies, the dissociation energies calculated by DFT were used as input values. The frequency factors entering the $G_{N}$ parameters were estimated from vapor pressure data of bulk platinum, and the instrumental flight times of the clusters were used. With this information, the intensity ratio of two consecutive sizes, $I_{N} / I_{N+1}$, can be calculated and compared directly with the measured values. This comparison is presented in panel (b) of the figure. The very good agreement between the modeled and measured ion intensity ratios confirms the quality of the quantum chemical calculations of the clusters' dissociation energies. Another, more direct, experimental check of the relation Eq. (33) has been made on sodium clusters with a comparison of two independent experimental determinations of binding energies of medium size clusters [87]. 
(a)

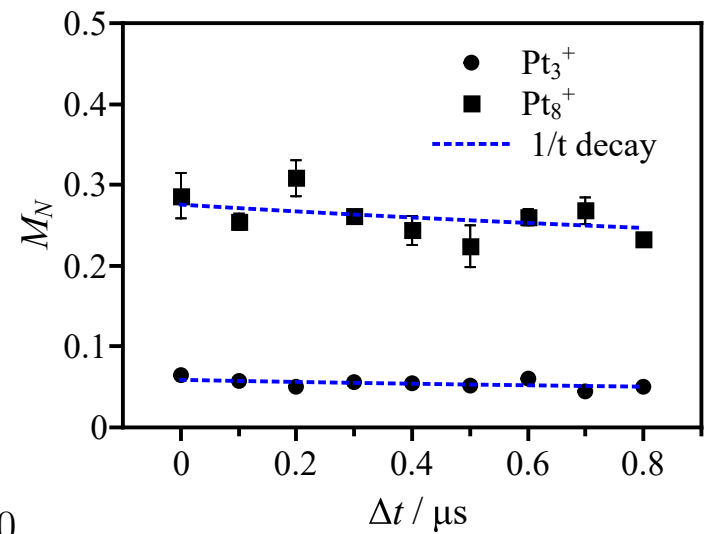

(b)

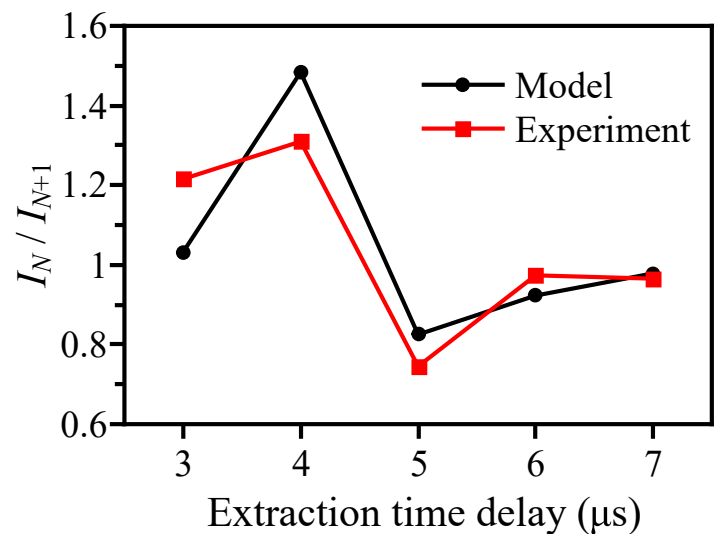

Figure 15: (a) Metastable fraction $\left(M_{N}\right)$ versus delay time $(\Delta t)$ of extraction for the photoexcited clusters $\mathrm{Pt}_{3}^{+}$and $\mathrm{Pt}_{8}^{+}$. A $1 / t$ law decay is assumed in the continuous line. As in Fig. 11, an instrumental time has been added to the abscissa to get the total value of the extraction delay time. (b) Comparison of cluster intensity ratios $I_{N} / I_{N+1}$ obtained mass spectrometrically (experiment) with those calculated from DFT values for dissociation energies and Eq. (33). Ref. [65] - Reproduced by permission of the PCCP Owner Societies.

\subsection{Radiative cooling and abundances}

The activation energy-abundance relation given in Eq. (33) is closely related to the power law decay, and when the latter is modified by radiative quenching, changes will also arise for abundances. If we restrict ourselves to the small photon energy cases, it is possible to find this effect by considering the effective time defined in Eq. (22). The integrand in the calculation of the rate in Eq. (23) gives the survival probability as a function of energy for a cluster excited with a broad energy distribution. This defines the abundances, as discussed in detail in $[73,80]$. The abundances in the presence of radiation are then found by the simple substitution of the time in Eq. (33) by the (size dependent) effective time;

$$
t \rightarrow \tau_{N} \equiv \frac{1}{\mathrm{w}_{N}}\left(1-\mathrm{e}^{-\mathrm{w}_{N} t_{N}}\right) .
$$

The instrumental time $t_{N}$ is only weakly size dependent, and is only mentioned for completeness. $\tau_{N}$ is shorter than $t_{N}$, and less fragmentation is therefore going to occur in the presence of radiation, in agreement with expectations.

The derivation of the abundances leading to Eq. (33) included two values of the Gspann parameter, one for each of the clusters involved in a decay, $N$ and $N+1$. In the non-radiative case, these two quantities will have practically identical values. For the 
radiative case, inserting the redefined and size-dependent time gives a formula similar to Eq. (33) with the substitution

$$
G_{N} \equiv \ln \left(\omega_{N} t_{N}\right) \rightarrow \ln \left(\omega_{N} \tau_{N}\right)
$$

with the size-dependence introduced by $\tau_{N}$. The resulting abundances become

$$
I_{N} \propto \frac{1}{2}\left(D_{N}^{\prime}+D_{N+1}^{\prime}\right)+C_{v, N}\left(\frac{D_{N}^{\prime}}{G_{N}}-\frac{D_{N+1}^{\prime}}{G_{N+1}}\right)
$$

The effect of radiation is to modify the denominators of the two terms in the last bracket with factors that are generally size dependent. One must expect large relative size variations of the radiative constants for electronically emitted photons, due to the effectively exponential suppression of the population of the high lying states with energy. Emission time scales can therefore vary by orders of magnitude. This difference is amplified by the heat capacity that multiplies the difference of these terms. In the harmonic approximation this factor is between $3 N-6$ and $3 N-9$.

An example of this analysis is presented in Fig. 16 for boron clusters. Using the method of delayed extraction of ions after laser excitation in the initial accelerating stage of a time-of-flight mass spectrometer, rates of photon emission were obtained for the small cationic boron clusters $\mathrm{B}_{N}^{+}(N=8-18)$. These rates are presented in panel (a) of the figure, showing that all the clusters in the investigated size range decay radiatively, although with very size dependent rates. The ratio of dissociation energies, $D_{N} / D_{N+1}$, can be extracted from mass spectra using Eq. (36) with a numerical solution of the difference equation. For comparison, the inversion has also been performed using the physical time $t$. Both cases are presented in panel (b) of the figure (blue triangles without radiation and black squares with it). As seen, both analyses give very similar results for the larger sizes, where the values of $k_{p}$ are small compared to the reciprocal initial acceleration time in the mass spectrometer. However, for the sizes $N=10$ and 11, where rates of photon emission are much higher, the radiative and non-radiative analysis give very different results. Disregarding thermal radiation, we would erroneously conclude that $\mathrm{B}_{11}^{+}$is much more stable than $\mathrm{B}_{12}^{+}$. Inclusion of the measured radiative cooling constants changes the pattern dramatically, as seen when comparing the two curves in panel (b) of Fig. 16. A comparison with the corresponding ratios using energies calculated with density functional theory (DFT) (ref. [88]) shows a remarkably good agreement with the values extracted from the mass spectra but only when radiation is taken into account as done here. 

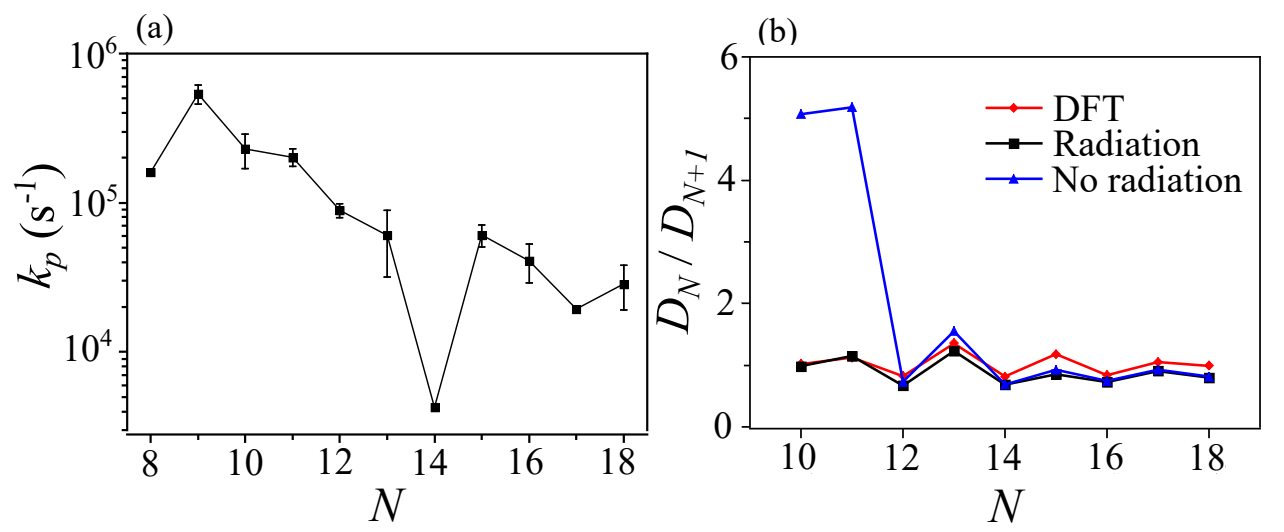

Figure 16: (a) Rate constants for photon emission from $\mathrm{B}_{N}^{+}$clusters. (b) Ratio of dissociation energies extracted from metastable fractions after photofragmentation. The analysis with and without radiative cooling is presented, together with the values calculated by DFT in Ref. [88]. Panels reprinted with permission from [89]. Copyright (2018) by the American Physical Society.

\section{Vibrational cooling versus recurrent fluorescence}

\subsection{Decays of carbon-based clusters}

So far the competition between fragmentation and radiation have been discussed with an emphasis on recurrent fluorescence. Vibrational radiative transitions is a well studied subject that can also give rise to the inverse process of IR heating of clusters and molecules in traps by absorption of ambient radiation [30, 31, 90-93]. In the vibrational cooling (VC) channel, a system decays by emitting photons in the far infrared (IR) range. Because the emitted photons have little energy, VC may be categorized as a process that occurs without any activation energy, although the quantum energy of the emitting modes may be significant relative to the temperature in interstellar space, for example. Vibrational transitions have small oscillator strengths and IR emission is therefore slow, with time constants on the order of tens of milliseconds or longer, as those seen for instance on the carbon cluster anions $\mathrm{C}_{5}^{-}$and $\mathrm{C}_{7}^{-}[94,95]$.

A striking example of the contrast between vibrational cooling and recurrent fluorescence are the decay times of laser excited $\mathrm{C}_{6} \mathrm{H}^{-}$and $\mathrm{C}_{6}^{-}$clusters [96]. The decays of both clusters were investigated in an electrostatic ion storage ring, in which neutralization yields were measured as a function of storage time after excitation with the third-harmonic of a nanosecond Nd:YAG laser $(355 \mathrm{~nm}, 3.49 \mathrm{eV})$. The neutralization rates were measured delayed by one half turn in the storage ring, corresponding to a time of $15 \mu \mathrm{s}$, thus elimi- 
nating direct processes. The photon energy was below the electron detachment thresholds of both clusters, and emission therefore required the presence of internal energies to reach the electron affinity. Combined with the delayed detection, this ensured that the emission was thermal.

The time profile of the decays are shown for different storage times, i.e. times before the laser was fired, in Fig. 17, in panels (a) and (b) for $\mathrm{C}_{6} \mathrm{H}^{-}$and $\mathrm{C}_{6}^{-}$, respectively [96]. The different colors represent different laser firing times, relative to $t=0$ when clusters were produced. It is obvious from the figure that the decays of the two clusters are very different. Whereas $\mathrm{C}_{6} \mathrm{H}^{-}$decays are quenched on a time scale of $1 \mathrm{~ms}$ or longer, the quenching of $\mathrm{C}_{6}^{-}$decays proceed significantly faster than the $30 \mu$ s circulation period in the ring.

The difference between the decays is directly related to the electronic structures of the two clusters. The first excited electronic state of $\mathrm{C}_{6} \mathrm{H}^{-}$has an energy of $3.77 \mathrm{eV}$. The population at the detachment threshold energy is unobservably low, less than $10^{-12}$, and recurrent fluorescence is consequently absent for $\mathrm{C}_{6} \mathrm{H}^{-}$. In contrast, $\mathrm{C}_{6}^{-}$has several states below $3 \mathrm{eV}(1.16 \mathrm{eV}, 1.32 \mathrm{eV}, 2.04 \mathrm{eV}$ and $2.49 \mathrm{eV})$, of which all except the $1.32 \mathrm{eV}$ state have optically allowed dipole transitions. The population of these states are calculated to $0.035,0.0013$ and $2.8 \times 10^{-5}$ for the states at $1.16 \mathrm{eV}, 2.04 \mathrm{eV}$ and $2.79 \mathrm{eV}$. Multiplied by the relatively large Einstein $A$-coefficients associated with electronic transitions, these populations give recurrent fluorescence rates on the order of $10^{4} \mathrm{~s}^{-1}$, in good agreement with experiments.

Rates of this magnitude not only contrast strongly with those of $\mathrm{C}_{6} \mathrm{H}^{-}$, but also to those of the cluster $\mathrm{C}_{5}^{-}$and $\mathrm{C}_{7}^{-}$. These are best described as cooling by $\mathrm{VC}$, in the tens of $\mathrm{ms}$ time range. An oscillatory pattern then appears with $\mathrm{RF}$ cooling for $\mathrm{C}_{4}^{-}[69,70]$, $\mathrm{VC}$ for $\mathrm{C}_{5}^{-}$[94], RF cooling for $\mathrm{C}_{6}^{-}$[70, 96-98], and $\mathrm{VC}$ for $\mathrm{C}_{7}^{-}$[95].

Experiments on positively charged $\mathrm{C}_{N}^{+}$clusters, in contrast, indicate $\mathrm{RF}$ cooling for all the non-fullerene sizes measured, $N=8,10,13-16$ [68], with rate constants scattered around $10^{4} \mathrm{~s}^{-1}$. The consistently higher rates for these clusters may be caused by the higher internal energies per degree of freedom of these species compared with the anions. Carbon dissociation energies are significantly higher than electron affinities, and these two activation energies determine the internal energies, or the effective temperatures, of the clusters. Although the tendency for carbon cations to radiate electronically is therefore not surprising, the numerical values of the radiative constants of the carbon cations in [68] are not well understood theoretically at the time of writing.

An important example of recurrent fluorescence is the cooling of anthracene, $\left(\mathrm{C}_{14} \mathrm{H}_{10}\right)^{+}$. Polycyclic aromatic hydrocarbons (PAHs) are common molecules in the interstellar medium 


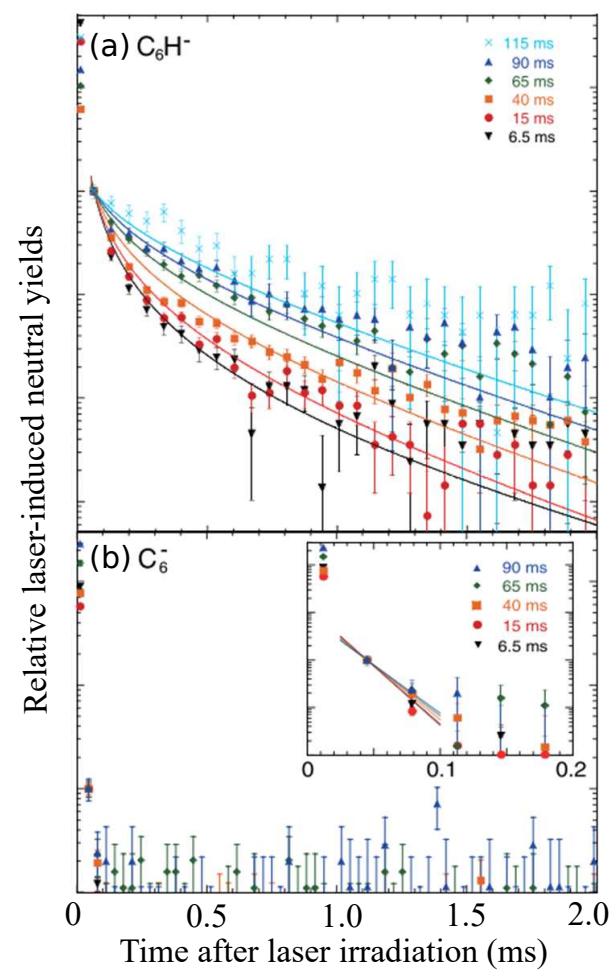

Figure 17: Storage time dependence of the decay profiles for (a) $\mathrm{C}_{6} \mathrm{H}^{-}$and (b) $\mathrm{C}_{6}^{-}$. The different colors represent decay profiles after excitation at different storage times, showing that the difference in cooling rates of $\mathrm{C}_{6} \mathrm{H}^{-}$and $\mathrm{C}_{6}^{-}$are not due to the initial cluster temperature before photoexcitation. Reprinted with permission from [96].

Copyright (2014) by the American Physical Society.

(ISM), which become highly excited by the UV radiation emitted by nearby stars. The cooling mechanisms of PAHs is an important factor determining the dynamics of the ISM, which will provide raw material for the formation of new planets and next generation stars [99]. Many PAHs have been detected in the ISM, among them anthracene, discovered in 2004 in neutral form [100], and in 2010 as a cation [101]. Gas phase experiments performed in a storage ring device, mimicking the conditions encountered by this molecule in the outer space, have shown that a beam of $\left(\mathrm{C}_{14} \mathrm{H}_{10}\right)^{+}$cools down radiatively at rates of around $200 \mathrm{~s}^{-1}$ upon laser excitation. These rates are two orders of magnitude higher than those expected from vibrational transitions, thus suggesting transitions of electronic nature via the RF mechanism [102-105].

Another molecule known to be present in the ISM is $\mathrm{C}_{60}[75,76,106]$. Gas phase experiments have shown that radiative cooling also proceeds via $\mathrm{RF}$ for a range of smaller fullerenes by experiments that could be performed in single pass mass spectrometers [19] 
for cations, and in a storage ring for the anions [20, 107]. The emitted power at the cross-over time from the electron emission to the radiative cooling regime is between $10^{2}$ and $10^{3} \mathrm{eV} / \mathrm{s}$, with a growing trend from the smallest to the largest molecules. These emission rates have been successfully modeled with a semiclassical description of the surface plasmon resonance [108], where the width of the resonance extends so far into the low energy regime that the otherwise very high electronic transition energies come into reach of thermal excitation.

A clear demonstration of the effect on the temperature generated by binding energies is provided by the results on the radiative cooling on naphtalene cations, $\mathrm{C}_{10} \mathrm{H}_{8}^{+}$, reported in [109] and [110]. The first paper gives measured quenching rates for the cationic molecule that are assigned to $\mathrm{RF}$, whereas the second paper, on the dimer cluster $\left(\mathrm{C}_{10} \mathrm{H}_{8}\right)_{2}^{+}$, reports a much slower cooling rate, consistent with vibrational cooling. The reason for the difference is the large difference in binding energies of the two species. The isolated molecule requires several $\mathrm{eV}$ to activate the lowest dissociation channel, which is a molecular dissociation, and the molecule is therefore able to support a fairly large effective temperature. The dimer, in contrast, is bound by relatively weak van der Waals forces that give a binding energy on the order of $0.5 \mathrm{eV}$. The dimer can only survive at lower temperatures, effectively reducing the population of electronically excited states to a vanishing value.

\subsection{Radiative cooling rates of metal and semi-metal clusters}

The radiative cooling of mass selected metal cluster cations of different elements have been investigated both in electrostatic storage rings/traps and in time-of-flight mass spectrometers, such as the one depicted in Fig. 9. Metals are of interest for their potential applications and because a large amount of valence electron oscillator strength resides in the surface plasmon resonance. This resonance is frequently found in the visible part of the spectrum, occasionally with strong tails to both sides, which can bring it into reach of thermal excitations, as for the fullerenes.

The radiative cooling measured with single pass mass spectrometers have mainly been on cationic clusters, although a positive charge is not a restriction in principle. At present, five metal or semi-metal cluster systems have been investigated with the delayed extraction/metastable fraction technique described previously, viz. $\mathrm{Nb}_{N}^{+}$[111], $\mathrm{Si}_{N}^{+}[67], \mathrm{B}_{N}^{+}$ [89], $\mathrm{Au}_{N}^{+}$[43] and $\mathrm{Pt}_{N}^{+}$[65], all of them using the same experimental setup and similar experimental conditions. This allows a direct comparison of the emission rates for the different clusters.

For $\mathrm{Pt}_{N}^{+}(N=3-8)$ no signs of radiative cooling are seen within the time scale of 
the experiment, and metastable fragmentation yields can be well fitted by an unmodified power law (see Fig. 15a). All the four other cluster species mentioned radiate, in some cases with very high rates and distinct size-dependences. Measured rate constant for the species not already discussed, assigned to single photon quenching, are shown in Fig. 18.

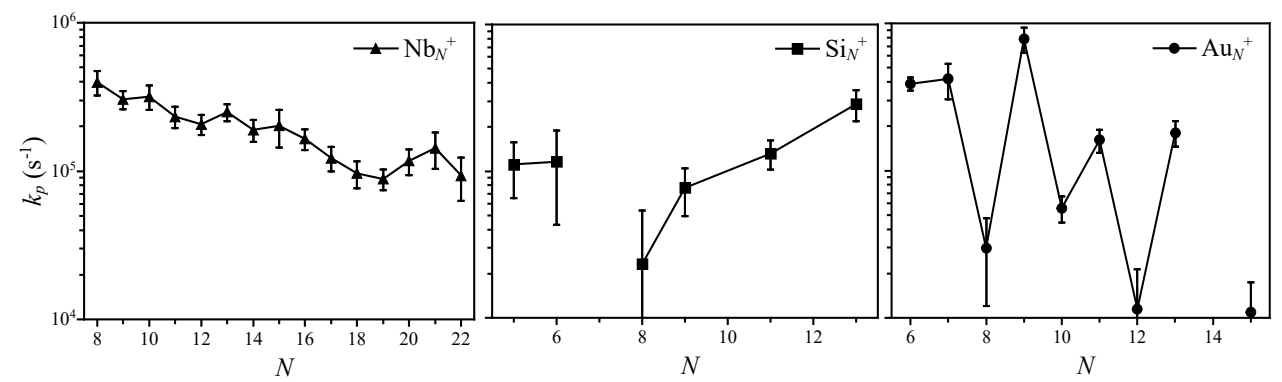

Figure 18: Rates of photon emission of $\mathrm{Nb}_{N}^{+}$(Reprinted from [111], with the permission of AIP Publishing), $\mathrm{Si}_{N}^{+}$(Reprinted from [67], with the permission of AIP Publishing) and $\mathrm{Au}_{N}^{+}$clusters, measured in a reflectron time-of-flight mass spectrometer (Reprinted with permission from [43]. Copyright (2017) by the American Physical Society).

The rates of $\mathrm{Nb}_{N}^{+}$show an overall decrease with cluster size, within the size range $N=8-22$, without much size-to-size variation, and with values between $10^{5} \mathrm{~s}^{-1}$ and $4 \times 10^{5} \mathrm{~s}^{-1}$. In $\mathrm{Si}_{N}^{+}, k_{p}$ is consistent with zero for $\mathrm{Si}_{7}^{+}$, after which values increase up to $3 \cdot 10^{5} \mathrm{~s}^{-1}$ for $\mathrm{Si}_{13}^{+}$. The boron data show radiation, as already witnessed by Fig. 16 . For $\mathrm{B}_{N}^{+}$a maximum in $k_{p}$ is observed for $\mathrm{B}_{9}^{+}$, with the high value of $5.4 \cdot 10^{5} \mathrm{~s}^{-1}$. Above this size, the rates tend to decrease monotonically except for a pronounced drop at $\mathrm{B}_{14}^{+}$, which has a rate consistent with zero. The final case is $\mathrm{Au}_{N}^{+}$, showing the most interesting size-dependence. Within the size range $N=6-15, k_{p}$ presents a pronounced odd-even oscillation, with higher values for clusters composed of an odd-number of atoms. These clusters have an even number of electrons and will have a higher than average stability. The highest rate constant for the measure sizes is seen for $\mathrm{Au}_{9}^{+}$, with the value of $8 \cdot 10^{5}$ $\mathrm{s}^{-1}$. A potential cause for this high radiation rate, and those of other gold clusters with even valence electron numbers is the high binding energy of these clusters. However, an analysis of the influence of the higher stability of even-electron numbered gold clusters on the radiative time constants has shown that only a small amount of the very strong radiative odd-even effect can be explained by this factor [43].

The very high rates obtained for several of the sizes of the four metal and semi-metal cluster species shown in Figs. 16 and 18 point directly to recurrent fluorescence as the underlying physical mechanism responsible for photon emission. By the nature of RF, 
this requires the presence of sufficiently low-lying electronic states. The states must also have sufficient oscillator strength, as this enters into the $A$-coefficient (see Eq. (1)). The measured cooling constants puts constraints on a combination of these two quantities. An analysis of this question for the gold cluster cations is given in [43]. One result of the analysis is that the optically active excited states are limited in energy by the ThomasReiche-Kuhn sum rules to be below $2 \mathrm{eV}$, and most likely will be found below $1.5 \mathrm{eV}$.

Storage ring experiments on radiative cooling of metal clusters have focused on anions. At present aluminium [56, 64, 112, 113], copper [114-116], and silver [56, 117] have been studied. The clusters tend to be small, due to the characteristics of the frequently used cesium sputter sources. The long storage times possible in these devices, recently expanded significantly by the introduction of cryogenic techniques, allows the determination of effects that manifest themselves on very long time scales, reaching into hours of

storage time. The lower limit of the time range is in the tens to hundreds of $\mu \mathrm{s}$, defined by the transfer time of the cluster from source to ring.

One effect identified in the experiments on copper clusters in [116] is the possible co-existence of isomeric structures caused by different and very high angular momenta of the clusters produced in the hot sources. As angular momentum is conserved, apart from the very slow rotational cooling, this allows the existence of populations of clusters that do not interconvert. The effect of the largest angular momenta, which can reach into hundreds of units of $\hbar$, is to stretch the rotating clusters. Such structure decay differently from the non-rotation species, both with respect to electron emission/atomic evaporation and to radiative cooling. For distributions of this type, decay curves that display radiation are sums of two radiatively quenched power laws. A remarkably good agreement with the experimental data for the copper cluster anions was obtained if the neutralization process is assumed to be quenched by the emission of large energy photons with cooling times that were fitted to in the range from $2.6 \mathrm{~ms}$ to $830 \mathrm{~ms}$ for the different cluster sizes. Such long cooling times are most likely due to vibrational cooling. In this case even vibrational cooling corresponds to the large photon energy regime due to the small size of the investigated clusters. The rotational population effect is still unexplored for $\mathrm{RF}$ radiation, but must be expected to be present for this also.

\section{Comments on the theoretical status}

Some comments on the theoretical state of the field is in place. The most common method to compute excited electronic states is time-dependent density functional theory (TDDFT), due to its favorable computational cost and accuracy when calculating optical 
absorption spectra in the visible range $[118,119]$. The predicted excited electronic states in gold clusters, however, are found above $2 \mathrm{eV}$ by this method [120], which is too high in energy to give the required populations, and with much too low oscillator strengths. A few theoretical studies predict low-lying electronic states in gold clusters, but these studies are restricted to the dimer, trimer, and tetramer clusters [121, 122]. These studies use complete active-space multiconfiguration self-consistent field theory with multireference configuration-interaction calculations in order to include electron correlations. Since such calculations are not available, to the best of our knowledge, in the size range investigated experimentally for the gold cations $(N=6-15)$, is it not possible to judge their predictability.

An important aspect of these theoretical modelings is that they are all based on geometric ground-state structures and do not consider the high internal energy of the clusters at the moment of photon emission. Geometry fluctuations, caused by the high temperature of the clusters, may well have a significant influence on the energy of the excited energy and hence its population, as considered in [123]. At present this effect is unexplored, and more theoretical work is required to judge its importance.

\section{Conclusions and outlook}

In this article we have presented a description of the radiative decay mechanisms available for an excited cluster in the gas phase. The rate at which radiation is emitted was shown to depend strongly on the cluster species. Both vibrational cooling and recurrent fluorescence were discussed, with an emphasis on the latter. In the former, several photons are emitted through vibrational transitions in the electronic ground state, whereas in the

latter, photons in the visible or near-IR spectral range are emitted from one or more thermally populated excited electronic state. Several examples of experiments performed with ion storage rings or single-pass time-of-flight mass spectrometers were discussed, showing the presence of radiative cooling from thermally excited electronic states in very different type of clusters, including small carbon-based clusters, fullerenes, semiconductor and metal clusters. Furthermore, the difference between a small and a large energy photon was derived, based on the effect emission has on a competing unimolecular rate. Also the effect of an active radiative cooling channel on the abundances of clusters was discussed. Radiative cooling can have important consequences for the determination of relative stability patterns of clusters by mass spectrometrical means, as demonstrated with an example. Theoretically, the attempts to reproduce the size- and element-dependent recurrent fluorescence rates of radiation of the many clusters discussed in this article by 
quantum chemical simulations have not been successful to date, and the quantum theoretical description is facing the challenge of catching up with the experimental development.

Finally we should mention that most studies of recurrent fluorescence have been performed in molecular beams. This is not a fundamental limitation; also ion traps and in principle neutral particle traps can be used for this purpose. An obvious extension will be the detection of the spectra, including time-resolved versions, of photons emitted by highly excited species.

\section{Acknowledgements}

This work is supported by the Research Foundation-Flanders (FWO/G0A05.19N) and by the KU Leuven Research Council (C14/18/073). PF acknowledges the FWO for a postdoctoral grant.

\section{References}

[1] O. Echt, K. Sattler, and E. Recknagel, Phys. Rev. Lett. 47, 1121 (1981).

[2] W. D. Knight, K. Clemenger, W. A. de Heer, W. A. Saunders, M. Y. Chou, and M. L. Cohen, Phys. Rev. Lett. 52, 2141 (1984).

[3] H. W. Kroto, J. R. Heath, S. C. O’Brien, R. F. Curl, and R. E. Smalley, Nature 318, 162 (1985).

[4] K. Selby, M. Vollmer, J. Masui, V. Kresin, W. A. de Heer, and W. D. Knight, Phys. Rev. B 40, 5417 (1989).

[5] J. Pedersen, J. Borggreen, P. Chowdhury, N. Kebaïli, L. Lundsberg-Nielsen, K. Lützenkirchen, M. B. Nielsen, and H. D. Rasmussen, Z. Phys. D 26, 281 (1993).

[6] B. Palpant, B. Prével, J. Lermé, E. Cottancin, M. Pellarin, M. Treilleux, A. Perez, J. Vialle, and M. Broyer, Phys. Rev. B 57, 1963 (1998).

[7] M. Schmidt, C. Ellert, W. Kronmüller, and H. Haberland, Phys. Rev. B 59, 10970 (1999).

[8] A. N. Gloess, H. Schneider, J. M. Weber, and M. M. Kappes, J. Chem. Phys. 128, 114312 (2008). 
[9] E. A. Rohlfing, D. M. Cox, and A. Kaldor, J. Chem. Phys. 81, 3322 (1984).

[10] E. Rohlfing, J. Chem. Phys. 89, 6103 (1988).

[11] U. Frenzel, A. Roggenkamp, and D. Kreisle, Chem. Phys. Lett. 240, 109 (1995).

[12] R. Mitzner and E. E. Campbell, J. Chem. Phys. 103, 2445 (1995).

[13] U. Frenzel, U. Kalmbach, D. Kreisle, and E. Recknagel, Surf. Rev. Lett. 3, 505 (1996).

[14] U. Frenzel, U. Hammer, H. Westje, and D. Kreisle, Z. Phys. D 40, 108 (1997).

[15] A. A. Vostrikov, D. Y. Dubov, and A. A. Agarkov, JETP Lett. 63, 963 (1996).

[16] A. Vostrikov, A. Agarkov, and D. Y. Dubov, Tech. Phys. 45, 915 (2000).

[17] P. Heszler, J. O. Carlsson, and P. Demirev, J. Chem. Phys. 107, 10440 (1997).

[18] A. A. Vostrikov, A. A. Agarkov, and Y. Dubov, Russ. Chem. B. 49, 276 (2000).

[19] K. Hansen and E. Campbell, J. Chem. Phys. 104, 5012 (1996).

[20] J. U. Andersen, C. Brink, P. Hvelplund, M. O. Larsson, B. B. Nielsen, and H. Shen, Phys. Rev. Lett. 77, 3991 (1996).

[21] S. Tomita, J. U. Andersen, C. Gottrup, P. Hvelplund, and U. Pedersen, Phys. Rev. Lett. 87, 073401 (2001).

[22] M. Hedén, K. Hansen, F. Jonsson, E. Rönnow, A. Gromov, A. Taninaka, H. Shinohara, and E. E. B. Campbell, J. Chem. Phys. 123, 044310 (2005).

[23] M. Yoshida, T. Furukawa, J. Matsumoto, H. Tanuma, T. Azuma, H. Shiromaru, and K. Hansen, in J. Phys. Conf. Ser. (IOP Publishing, 2017), vol. 875, p. 012017.

[24] Y. Ebara, T. Furukawa, J. Matsumoto, H. Tanuma, T. Azuma, H. Shiromaru, and K. Hansen, Phys. Rev. Lett. 117, 133004 (2016).

[25] A. Nitzan and J. Jortner, J. Chem. Phys. 71, 3524 (1979).

[26] J. Jortner, in Polycyclic Aromatic Hydrocarbons and Astrophysics, edited by M. D. Cohen (Buitterworth (London), 1971), pp. 389-419.

[27] A. Nitzan and J. Jortner, J. Chem. Phys. 56, 5200 (1972). 
[28] A. E. Bragg, J. R. Verlet, A. Kammrath, and D. M. Neumark, J. Chem. Phys. 121, 3515 (2004).

[29] A. Tramer, C. Jungen, and F. Lahmani, Energy Dissipation in Molecular Systems (Springer, 2005).

[30] R. C. Dunbar, J. Chem. Phys. 90, 7369 (1989).

[31] R. C. Dunbar, Mass Spectrom. Rev. 11, 309 (1992).

[32] S. Leach, in Polycyclic Aromatic Hydrocarbons and Astrophysics, edited by A. Léger, L. d'Hendecourt, and N. Boccara (D. Reidel Publishing Company, 1987), vol. 191 of NATO ASI Series, pp. 99-127, ISBN 978-94-010-8619-6.

[33] A. Léger, P. Boissel, and L. d'Hendecourt, Phys. Rev. Lett. 60, 921 (1988).

[34] V. Chernyy, R. Logemann, J. M. Bakker, and A. Kirilyuk, J. Chem. Phys. 145, 024313 (2016).

[35] W. Forst, Theory of Unimolecular Reactions (Academic Press (New York and London), 1973).

[36] T. Leisner, K. Athanassenas, O. Kandler, D. Kreisle, E. Recknagel, and O. Echt, MRS Proceedings 206, 259 (1990).

[37] P. Wurz and K. R. Lykke, J. Chem. Phys. 95, 7008 (1991).

[38] E. E. B. Campbell, G. Ulmer, and I. V. Hertel, Phys. Rev. Lett. 67 (1991).

[39] J. U. Andersen, E. Bonderup, and K. Hansen, J. Phys. B At. Mol. Opt. Phys. 35, R1 (2002).

[40] U. Näher, S. Bjørnholm, S. Frauendorf, F. Garcias, and C. Guet, Phys. Rep. 285, 245 (1997), ISSN 0370-1573.

[41] S. König, A. Jankowski, G. Marx, L. Schweikhard, and M. Wolfram, Phys. Rev. Lett. 120, 163001 (2018).

[42] K. Hansen, A. Herlert, L. Schweikhard, and M. Vogel, Phys. Rev. A 73, 063202 (2006).

[43] K. Hansen, P. Ferrari, E. Janssens, and P. Lievens, Phys. Rev. A 96, 022511 (2017). 
[44] C. Jackschath, I. Rabin, and W. Schulze, Ber. Bunsenges. Phys. Chem. 96, 1200 (1992).

[45] T. Beyer and D. F. Swinehart, Commun. ACM 16, 379 (1973).

[46] K. Hansen, Statistical Physics of Nanoparticles in the Gas Phase (Springer, 2018).

[47] K. Hansen and E. E. B. Campbell, Phys. Rev. E 58, 5477 (1998).

[48] L. J. Allamandola, A. G. G. M. Tielens, and J. R. Barker, Astrophys. J., Suppl. Ser. 71, 733 (1989).

[49] B. H. Bransden and C. J. Joachain, Physics of Atoms and Molecules (Prentice Hall, Harlow, 2003), 2nd edition.

[50] S. Jinno, T. Takao, K. Hanada, M. Goto, K. Okuno, H. Tanuma, T. Azuma, and H. Shiromaru, Nucl. Instrum. Meth. A 572, 568 (2007).

[51] S. P. Møller, Nucl. Instr. Meth. A 394, 281 (1997).

[52] L. H. Andersen, O. Heber, and D. Zajfman, J. Phys. B.: At. Mol. Opt. 37, 57 (2004).

[53] J. U. Andersen, C. Brink, P. Hvelplund, M. O. Larsson, and H. Shen, Z. Phys. D 40, 365 (1997).

[54] J. Lemaire, M. Heninger, R. Marx, and G. Mauclaire, Int. J. Mass Spectrom. 189, 93 (1999).

[55] C. Walther, G. Dietrich, W. Dostal, K. Hansen, S. Krückeberg, K. Lützenkirchen, and L. Schweikhard, Phys. Rev. Lett. 83, 3816 (1999).

[56] K. Hansen, J. U. Andersen, P. Hvelplund, S. Møller, U. Pedersen, and V. Petrunin, Phys. Rev. Lett. 87, 123401 (2001).

[57] K. Mehlig, K. Hansen, M. Hedén, A. Lassesson, A. Bulgakov, and E. E. B. Campbell, J. Chem. Phys. 120, 4281 (2004).

[58] N. Veldeman, E. Janssens, K. Hansen, J. De Haeck, R. E. Silverans, and P. Lievens, Faraday Discuss. 138, 147 (2008).

[59] K. Hansen and O. Echt, Phys. Rev. Lett. 78, 2337 (1997). 
[60] J. U. Andersen, E. Bonderup, K. Hansen, P. Hvelplund, B. Liu, U. Pedersen, and S. Tomita, Eur. Phys. J. D 24, 191 (2003).

[61] J. Fedor, K. Hansen, J. U. Andersen, and P. Hvelplund, Phys. Rev. Lett. 94, 113201 (2005).

[62] J. Rajput, L. Lammich, and L. H. Andersen, Phys. Rev. Lett. 100, 153001 (2008).

[63] J. Andersen, H. Cederquist, J. Forster, B. Huber, P. Hvelplund, J. Jensen, B. Liu, B. Manil, L. Maunoury, S. Brøndsted Nielsen, et al., Eur. Phys. J. D 25, 139 (2003).

[64] M. W. Froese, K. Blaum, F. Fellenberger, M. Grieser, M. Lange, F. Laux, S. Menk, D. A. Orlov, R. Repnow, T. Sieber, et al., Phys. Rev. A 83, 023202 (2011).

[65] P. Ferrari, K. Hansen, P. Lievens, and E. Janssens, Phys. Chem. Chem. Phys. 20, 29085 (2018).

[66] I. Katakuse, T. Ichihara, Y. Fujita, T. Matsuo, T. Sakurai, and H. Matsuda, Int. J. Mass. Spectrom. Ion Process. 67, 229 (1985).

[67] P. Ferrari, E. Janssens, P. Lievens, and K. Hansen, J. Chem. Phys. 143, 224313 (2015).

[68] F. Chen, N. Kono, R. Suzuki, T. Furukawa, H. Tanuma, P. Ferrari, T. Azuma, J. Matsumoto, H. Shiromaru, V. Zhaunerchyk, et al., Phys. Chem. Chem. Phys. 21, 1587 (2019).

[69] N. Kono, T. Furukawa, H. Tanuma, J. Matsumoto, H. Shiromaru, T. Azuma, K. Najafian, M. S. Pettersson, B. Dynefors, and K. Hansen, Phys. Chem. Chem. Phys. 17, 24732 (2015).

[70] N. Kono, R. Suzuki, T. Furukawa, J. Matsumoto, H. Tanuma, H. Shiromaru, T. Azuma, and K. Hansen, Phys. Rev. A 98, 063434 (2018).

[71] M. Abramowitz and I. Stegun, Handbook of Mathematical Functions (Dover Publications, New York, 1970), ISBN 486-61272-4.

[72] K. Hansen, H. Hohmann, R. Müller, and E. Campbell, J. Chem. Phys. 105, 6088 (1996).

[73] K. Hansen and P. Ferrari, Chin. J. Chem. Phys. 32, 162 (2019). 
[74] A. E. K. Sundén, M. Goto, J. Matsumoto, H. Shiromaru, H. Tanuma, T. Azuma, J. U. Andersen, S. E. Canton, and K. Hansen, Phys. Rev. Lett. 103, 143001 (2009).

[75] J. Cami, J. Bernard-Salas, E. Peeters, and S. E. Malek, Science 329, 1180 (2010).

[76] E. K. Campbell, M. Holz, D. Gerlich, and J. P. Maier, Nature 523, 322 (2015).

[77] M. A. Duncan, Rev. Sci. Instrum. 83, 041101 (2012).

[78] T. P. Martin, Phys. Rep. 273, 199 (1996).

[79] Y. Huttel, ed., Gas-Phase Synthesis of Nanoparticles (Wiley, VCH, 2017).

[80] K. Hansen and U. Näher, Phys. Rev. A 60, 1240 (1999).

[81] K. Hansen, P. Andersson, and E. Uggerud, J. Chem. Phys. 131, 124303 (2009).

[82] S. Bjørnholm and J. Borggreen, Philos. Mag. B 79, 1321 (1999).

[83] J. Borggreen, K. Hansen, F. Chandezon, T. Døssing, M. Elhajal, and O. Echt, Phys. Rev A 62, 013202 (2000).

[84] J. Stenfalk and K. Hansen, Eur. Phys. J. D 43, 101 (2007).

[85] J. Gspann, Z. Phys. D pp. 143-145 (1986).

[86] C. E. Klots, J. Chem. Phys. 90, 4470 (1989).

[87] J. Borggreen, K. Hansen, F. Chandezon, T. Døssing, M. Elhajal, and O. Echt, Phys. Rev. A 62, 013202 (2000).

[88] T. B. Tai, N. M. Tam, and M. T. Nguyen, Theor. Chem. Acc. 131, 1241 (2012).

[89] P. Ferrari, J. Vanbuel, K. Hansen, P. Lievens, E. Janssens, and A. Fielicke, Phys. Rev. A 98, 012501 (2018).

[90] T. Schindler, C. Berg, G. Niedner-Schatteburg, and V. Bondybey, Chem. Phys. Lett. 250, 301 (1996).

[91] G. Niedner-Schatteburg and V. E. Bondybey, Chem. Rev. 100, 4059 (2000).

[92] Y. Toker, I. Rahinov, D. Schwalm, U. Even, O. Heber, M. L. Rappaport, D. Strasser, and D. Zajfman, Phys. Rev. A 86, 023202 (2012). 
[93] P. Schmitt-Kopplin and B. Kanawati, Fundamentals and Applications of Fourier Transform Mass Spectrometry (Elsevier, Netherlands, 2019), 1st edition.

[94] M. Goto, A. Sundén, H. Shiromaru, J. Matsumoto, H. Tanuma, T. Azuma, and K. Hansen, J. Chem. Phys. 139, 054306 (2013).

[95] K. Najafian, M. S. Pettersson, B. Dynefors, H. Shiromaru, J. Matsumoto, H. Tanuma, T. Furukawa, T. Azuma, and K. Hansen, J. Chem. Phys. 140, 104311 (2014).

[96] G. Ito, T. Furukawa, H. Tanuma, J. Matsumoto, H. Shiromaru, T. Majima, M. Goto, T. Azuma, and K. Hansen, Phys. Rev. Lett. 112, 183001 (2014).

[97] V. Chandrasekaran, B. Kafle, A. Prabhakaran, O. Heber, M. Rappaport, H. Rubinstein, D. Schwalm, Y. Toker, and D. Zajfman, J. Phys. Chem. Lett. 5, 4078 (2014).

[98] V. Chandrasekaran, A. Prabhakaran, B. Kafle, H. Rubinstein, O. Heber, M. Rappaport, Y. Toker, and D. Zajfman, J. Chem. Phys. 146, 094302 (2017).

[99] A. Candian, J. Zhen, and A. G. Tielens, Phys. Today 71, 38 (2018).

[100] U. P. Vijh, A. N. Witt, and K. D. Gordon, Astrophys. J. 606, L65 (2004).

[101] S. Iglesias-Groth, A. Manchado, R. Rebolo, J. González Hernández, D. GarcíaHernández, and D. Lambert, Mon. Not. R. Astron. Soc. 407, 2157 (2010).

[102] S. Martin, J. Bernard, R. Brédy, B. Concina, C. Joblin, M. Ji, C. Ortega, and L. Chen, Phys. Rev. Lett. 110, 063003 (2013).

[103] M. Ji, J. Bernard, L. Chen, R. Brédy, C. Ortéga, C. Joblin, A. Cassimi, and S. Martin, J. Chem. Phys. 146, 044301 (2017).

[104] S. Martin, M. Ji, J. Bernard, R. Brédy, B. Concina, A. Allouche, C. Joblin, C. Ortega, G. Montagne, A. Cassimi, et al., Phys. Rev A 92 (2015).

[105] S. Martin, L. Chen, A. Al-Mogeeth, and J. Bernard, Phys. Rev. A 99, 012712 (2019).

[106] B. Foing and P. Ehrenfreund, Nature 369, 296 (1994).

[107] J. Andersen, C. Gottrup, K. Hansen, P. Hvelplund, and M. Larsson, Eur. Phys. J. D 17, 189 (2001). 
[108] J. Andersen and E. Bonderup, Eur. Phys. J. D 11, 413 (2000).

[109] J. Bernard, A. Al-Mogeeth, A.-R. Allouche, L. Chen, G. Montagne, and S. Martin, J. Chem. Phys. 150, 054303 (2019).

[110] S. Martin, J. Matsumoto, N. Kono, M.-C. Ji, R. Brédy, J. Bernard, A. Cassimi, and L. Chen, Nucl. Instrum. Meth. B 408, 209 (2017).

[111] K. Hansen, Y. Li, V. Kaydashev, and E. Janssens, J. Chem. Phys. 141, 024302 (2014).

[112] Y. Toker, O. Aviv, M. Eritt, M. L. Rappaport, O. Heber, D. Schwalm, and D. Zajfman, Phys. Rev. A 76, 053201 (2007).

[113] M. Lange, M. W. Froese, S. Menk, D. Bing, F. Fellenberger, M. Grieser, F. Laux, D. A. Orlov, R. Repnow, T. Sieber, et al., New J. Phys. 14, 065007 (2012).

[114] M. H. Stockett, M. Kamińska, R. F. Nascimento, E. K. Anderson, M. Gatchell, K. Hansen, H. Zettergren, H. T. Schmidt, and H. Cederquist, J. Phys. Conf. Ser. 635, 072090 (2015).

[115] C. Breitenfeldt, K. Blaum, M. W. Froese, S. George, G. Guzmán-Ramírez, M. Lange, S. Menk, L. Schweikhard, and A. Wolf, Phys. Rev. A 94, 033407 (2016).

[116] K. Hansen, M. H. Stockett, M. Kaminska, R. F. Nascimento, E. K. Anderson, M. Gatchell, K. C. Chartkunchand, G. Eklund, H. Zettergren, H. T. Schmidt, et al., Phys. Rev. A 95, 022511 (2017).

[117] E. K. Anderson, M. Kamińska, K. C. Chartkunchand, G. Eklund, M. Gatchell, K. Hansen, H. Zettergren, H. Cederquist, and H. T. Schmidt, Phys. Rev. A 98, 022705 (2018).

[118] A. Shayeghi, C. Heard, R. Johnston, and R. Schäfer, J. Chem. Phys. 140, 054312 (2014).

[119] V. Kaydashev, P. Ferrari, C. Heard, E. Janssens, R. L. Johnston, and P. Lievens, Part. Part. Syst. Char. 33, 364 (2016).

[120] J. V. Koppen, M. Hapka, M. M. Szczesniak, and G. Chalasinski, J. Chem. Phys. 137, $114302(2012)$.

[121] K. Balasubramanian, J. Mol. Struct. 202, 291 (1989). 
[122] K. Balasubramanian and K. Das, Chem. Phys. Lett. 186, 577 (1991).

[123] V. V. Kresin and Y. N. Ovchinnikov, Phys. Rev. B 73, 115412 (2006). 